OPEN

SUBJECT AREAS:

QUANTUM INFORMATION

QUBITS

Received

24 June 2014

Accepted

1 December 2014

Published

19 December 2014

Correspondence and requests for materials should be addressed to

S.Z. (szhang@ybu. edu.cn)

\title{
Preparation of three-dimensional
} entanglement for distant atoms in coupled cavities via atomic spontaneous emission and cavity decay

\author{
Shi-Lei Su ${ }^{1,2}$, Xiao-Qiang Shao ${ }^{3,4}$, Hong-Fu Wang ${ }^{2}$ \& Shou Zhang ${ }^{1,2}$
}

\begin{abstract}
'Department of Physics, Harbin Institute of Technology, Harbin 150001, China, ${ }^{2}$ Department of Physics, College of Science, YanBian University, Yanji, Jilin 133002, China, ${ }^{3}$ Centre for Quantum Sciences and School of Physics, Northeast Normal University, Changchun 130024, China, ${ }^{4}$ Centre for Quantum Technologies, National University of Singapore, 3 Science Drive 2, Singapore, 117543.
\end{abstract}

We propose a dissipative scheme to prepare a three-dimensional entangled state for two atoms trapped in separate coupled cavities. Our work shows that both atomic spontaneous emission and cavity decay, which are two typical obstacles in unitary-dynamics-based schemes, are no longer detrimental, but necessary for three-dimensional entangled state preparation without specifying initial state and controlling the evolution time precisely. Final numerical simulation with one group of experimental parameters indicates that the performance of our scheme could be better than the unitary-dynamics-based scheme.

$\mathrm{t}$ is well known that the dissipation induced by the environment is inevitable in the development of quantum science and technology. For a long time, dissipation has been regarded as a major obstacle to quantum information technology. Generally, there are two common methods to deal with the decoherence. One is quantum error correction ${ }^{1-3}$, which relies on high-fidelity gates for detecting as well as correcting errors, and the other is to encode the qubits into a decoherencefree subspace (DFS) in multipartite system ${ }^{4-7}$ by utilizing coupling symmetry between system and environment. Fundamentally different from the former methods, using dissipation as powerful resource has special merits since it is used to create entanglement ${ }^{8-23}$ or realize spin squeezing state $^{24-26}$ rather than destroy entanglement. Particularly, Kastoryano et al. considered a dissipative scheme for preparing a maximally entangled state of two $\Lambda$ atoms in a high finesse optical cavity without requirement of state initialization $^{13}$. And Shen et al. generalized the scheme to prepare distributed entanglement via dissipation ${ }^{14,15}$. Besides, Reitor et al. presented a scheme for the dissipative preparation of an entangled steady state of two superconducting qubits ${ }^{16}$. These schemes show that cavity decay is no longer undesirable, but plays a positive role in state preparation. Nevertheless, the atomic spontaneous emission would decrease the performance of these schemes. Recently, Shao et al. proposed a dissipative scheme which shows that atomic spontaneous emission also has the ability to be used to prepare the entangled state ${ }^{22}$.

Coupled cavity model provides an essential tool for distributed quantum information processing and has been studied both theoretically ${ }^{27-34}$ and experimentally ${ }^{35}$. Most of the coupled-cavity-system-based scheme focus on the coherent unitary dynamics that requires time control and state initialization. Motivated by Ref. 13, Shen et al. designed a dissipative scheme to prepare steady-state entanglement in coupled cavities which requires neither definite initial states nor precise time control ${ }^{14}$.

High-dimensional entangled states have attracted more and more attentions owing to the fact that they can enhance the security of quantum key distribution ${ }^{36,37}$ and violate the local realism more strongly than the twodimensional entanglement ${ }^{38}$. How to realize high-dimensional entanglement has been researched in the fields of linear optics experimentally by utilizing the spatial modes of photons carrying orbital angular momentum information ${ }^{39,40}$ and of cavity quantum electrodynamics (QED) theoretically through the unitary dynamics ${ }^{41-44}$.

As is well known to us, atomic spontaneous emission and cavity decay are two typical decoherence factors, which would decrease the feasibility of the unitary-dynamics-based scheme. The previous works show that dissipative schemes could use either cavity decay or spontaneous emission separately to prepare entanglement, but when one of the factors exerts positive effects on state preparation, the other may decrease the overall 
performance of the scheme. Thus, using both decoherence factors to prepare entanglement has unique characteristics. Although cooling schemes meet this goal, more classical fields are required to resonantly drive the undesired state to single-excitation subspace which would decay to the desired state probably ${ }^{45,46}$. In this paper, we propose a dissipative scheme, which makes full use of unitary dynamics provided by microwave field and dissipative factors originating from spontaneous emission and cavity decay, to prepare the three-dimensional entangled state in coupled cavities. In order to know more clearly about the effect of each dissipative factor, we first consider the system without cavity decay, and then consider it without atomic spontaneous emission. The analytical and numerical results show that both cavity decay and atomic spontaneous emission are capable of being useful resources for entanglement preparation. However, the cavity-decay-based case is not as ideal as the spontaneous-emission-based case, which could be improved through adding quantum feedback control. Interestingly, conditions for achieving the dominant dissipative channels of the spontaneous-emission-based case are almost the same to that of the cavity-decay-based case, which could be satisfied at the same time. Therefore, it is possible to use both spontaneous emission and cavity decay to prepare entangled state simultaneously. There are several main characteristics of our scheme. (i) Our scheme is independent of the initial state and do not require precise time control. (ii) Both spontaneous emission and cavity decay could be utilized for preparing the desired entangled state. (iii) With specific parameters extracted from the experiment, the fidelity of our scheme could be $97.24 \%$, which exceeds the values in the schemes ${ }^{42-44}$ based on the unitary dynamics.

\section{Results}

Basic model. Considering a system composed of two ${ }^{87} \mathrm{Rb}$ atoms trapped in bimode coupled cavities, as shown in Fig. 1. For the first (second) atom, an off-resonance $\pi$-polarized optical laser with detuning $\Delta$, Rabi frequency $\Omega_{1(2)}$ is applied to drive the transition $\left|e_{0}\right\rangle$ $\leftrightarrow\left|g_{a}\right\rangle\left(\left|e_{L}\right\rangle \leftrightarrow\left|g_{L}\right\rangle\right.$ and $\left|e_{R}\right\rangle \leftrightarrow\left|g_{R}\right\rangle$, respectively). The cavity modes $a_{L 1(L 2)}$ and $a_{R 1(R 2)}$ are coupled to the transitions $\left|e_{0(R)}\right\rangle \leftrightarrow\left|g_{L(0)}\right\rangle$ and $\left|e_{0(L)}\right\rangle \leftrightarrow\left|g_{R(0)}\right\rangle$ with detuning $\Delta-\delta$, coupling strength $g_{L}$ and $g_{R}$, respectively. A microwave field with Rabi frequency $\omega_{1}$ is introduced to realize the transitions $\left|g_{L}\right\rangle \leftrightarrow\left|g_{a}\right\rangle$ and $\left|g_{R}\right\rangle \leftrightarrow\left|g_{a}\right\rangle$ of atom 1 . Besides, degenerate Raman coupling process which could realize the coupling between the degenerate states ${ }^{48-52}$ is introduced to achieve the transitions $\left|g_{L}\right\rangle \leftrightarrow\left|g_{0}\right\rangle$ and $\left|g_{R}\right\rangle \leftrightarrow\left|g_{0}\right\rangle$ of atom 2 with effective coupling strength $\omega_{2}$. In addition, if an external magnetic field is introduced for the atom 2 to split $\left|g_{L}\right\rangle$ and $\left|g_{R}\right\rangle$ from $\left|g_{0}\right\rangle$ with a certain energy difference ${ }^{53}$, a microwave field with the energy matching the difference could also be used to achieve the couplings $\left|g_{L}\right\rangle \leftrightarrow\left|g_{0}\right\rangle$ and $\left|g_{R}\right\rangle \leftrightarrow\left|g_{0}\right\rangle$.

Under the rotating-wave approximation, the Hamiltonian of the whole system in a rotating frame reads $H=H_{0}+H_{g}+V_{+}+V_{-}$, where

$$
\begin{gathered}
H_{0}=\delta\left(\hat{a}_{L 1}^{\dagger} \hat{a}_{L 1}+\hat{a}_{L 2}^{\dagger} \hat{a}_{L 2}+\hat{a}_{R 1}^{\dagger} \hat{a}_{R 1}+\hat{a}_{R 2}^{\dagger} \hat{a}_{R 2}\right)+\left[g_{L}\left|g_{L}\right\rangle_{11}\left\langle e_{0}\left|\hat{a}_{L 1}^{\dagger}+g_{L}\right| g_{0}\right\rangle_{22}\left\langle e_{R}\right| \hat{a}_{L 2}^{\dagger}\right. \\
\left.+g_{R}\left|g_{R}\right\rangle_{11}\left\langle e_{0}\left|\hat{a}_{R 1}^{\dagger}+g_{R}\right| g_{0}\right\rangle_{22}\left\langle e_{L}\right| \hat{a}_{R 2}^{\dagger}+\text { H.c. }\right]+\Delta\left(\left|e_{0}\right\rangle_{11}\left\langle e_{0}|+| e_{L}\right\rangle_{22}\left\langle e_{L}|+| e_{R}\right\rangle_{22}\left\langle e_{R}\right|\right)(1) \\
+J_{L}\left(\hat{a}_{L 1}^{\dagger} \hat{a}_{L 2}+\text { H.c. }\right)+J_{R}\left(\hat{a}_{R 1}^{\dagger} \hat{a}_{R 2}+\text { H.c. }\right) \\
H_{g}=\omega_{1}\left(\left|g_{L}\right\rangle_{11}\left\langle g_{a}|+| g_{R}\right\rangle_{11}\left\langle g_{a}\right|+\text { H.c. }\right) \\
+\omega_{2}\left(\left|g_{L}\right\rangle_{22}\left\langle g_{0}|+| g_{R}\right\rangle_{22}\left\langle g_{0}\right|+\text { H.c. }\right) \\
V_{+}=\Omega_{1}\left|e_{0}\right\rangle_{11}\left\langle g_{a}\right|+\Omega_{2}\left(\left|e_{L}\right\rangle_{22}\left\langle g_{L}|+| e_{R}\right\rangle_{22}\left\langle g_{R}\right|\right) \\
V_{-}=V_{+}^{\dagger}
\end{gathered}
$$

in which $\hat{a}_{L i}$ and $\hat{a}_{R i}$ are the cavity operators in cavity $i(i=1,2) . J_{L(R)}$ denotes the photon-hopping strength between two coupled cavities. By introducing four delocalized bosonic modes $\hat{c}_{L 1}=\left(\hat{a}_{L i}-\hat{a}_{L 2}\right) / \sqrt{2}$, $\hat{c}_{L 2}=\left(\hat{a}_{L 1}+\hat{a}_{L 2}\right) / \sqrt{2}, \quad \hat{c}_{R 1}=\left(\hat{a}_{R 1}-\hat{a}_{R 2}\right) / \sqrt{2}, \hat{c}_{R 2}=\left(\hat{a}_{R 1}+\hat{a}_{R 2}\right) / \sqrt{2}$, the Hamiltonian $H_{0}$ can be rewritten as

$$
\begin{aligned}
H_{0}= & \left(\delta-J_{L}\right) \hat{c}_{L 1}^{\dagger} \hat{c}_{L 1}+\left(\delta+J_{L}\right) \hat{c}_{L 2}^{\dagger} \hat{c}_{L 2}+\left(\delta-J_{R}\right) \hat{c}_{R 1}^{\dagger} \hat{c}_{R 1}+\left(\delta+J_{R}\right) \hat{c}_{R 2}^{\dagger} \hat{c}_{R 2} \\
& +\frac{g_{L}}{\sqrt{2}}\left[\left|g_{L}\right\rangle_{11}\left\langle e_{0}\left|\left(\hat{c}_{L 1}^{\dagger}+\hat{c}_{L 2}^{\dagger}\right)+\right| g_{0}\right\rangle_{22}\left\langle e_{R}\right|\left(\hat{c}_{L 2}^{\dagger}-\hat{c}_{L 1}^{\dagger}\right)+\text { H.c. }\right] \\
& +\frac{g_{R}}{\sqrt{2}}\left[\left|g_{R}\right\rangle_{11}\left\langle e_{0}\left|\left(\hat{c}_{R 1}^{\dagger}+\hat{c}_{R 2}^{\dagger}\right)+\right| g_{0}\right\rangle_{22}\left\langle e_{L}\right|\left(\hat{c}_{R 2}^{\dagger}-\hat{c}_{R 1}^{\dagger}\right)+\text { H.c. }\right] \\
& +\Delta\left(\left|e_{0}\right\rangle_{11}\left\langle e_{0}|+| e_{L}\right\rangle_{22}\left\langle e_{L}|+| e_{R}\right\rangle_{22}\left\langle e_{R}\right|\right) .
\end{aligned}
$$

For simplicity, we set $g_{L}=g_{R}=g, \Omega_{1}=\Omega_{2}=\Omega, J_{L}=J_{R}=J$ and $\omega_{1}=$ $-\omega_{2}=\omega$ in the following. The photon decay rate of cavity $i$ is denoted as $\kappa_{i}(i=1,2)$ (suppose two field modes in the same cavity have the same decay rate). The excited state of the first atom $\left|e_{0}\right\rangle$ spontaneously decays into ground states with branching rate $\gamma_{1} / 3$, while the states $\left|e_{L(R)}\right\rangle$ of the second atom are translated into $\left|g_{L(R)}\right\rangle$ and $\left|g_{0}\right\rangle$ with rate $\gamma_{2} / 2$. We assume $\kappa_{1}=\kappa_{2}=\kappa$ and $\gamma_{1}=\gamma_{2}=\gamma$ throughout this paper. Thus, the Lindblad operators associated with the cavity decay and spontaneous emission can be expressed as $L^{\kappa, c_{L 1}}=\sqrt{\kappa} \hat{c}_{L 1}, \quad L^{\kappa, c_{R 1}}=\sqrt{\kappa} \hat{c}_{R 1}, \quad L^{\kappa, c_{L 2}}=\sqrt{\kappa} \hat{c}_{L 2}, \quad L^{\kappa, c_{R 2}}=\sqrt{\kappa} \hat{c}_{R 2}$, $L^{\gamma_{1}, g_{L(a, R)}}=\sqrt{\gamma / 3}\left|g_{L(a, R)}\right\rangle_{11}\left\langle e_{0}\left|, \quad L^{\gamma_{2}, g_{L(0 L)}}=\sqrt{\gamma / 2}\right| g_{L(0)}\right\rangle_{22}\left\langle e_{L}\right| \quad$ and $L^{\gamma_{2}, g_{R(0 R)}}=\sqrt{\gamma / 2}\left|g_{R(0)}\right\rangle_{22}\left\langle e_{R}\right|$. Then, the dynamics of our system is

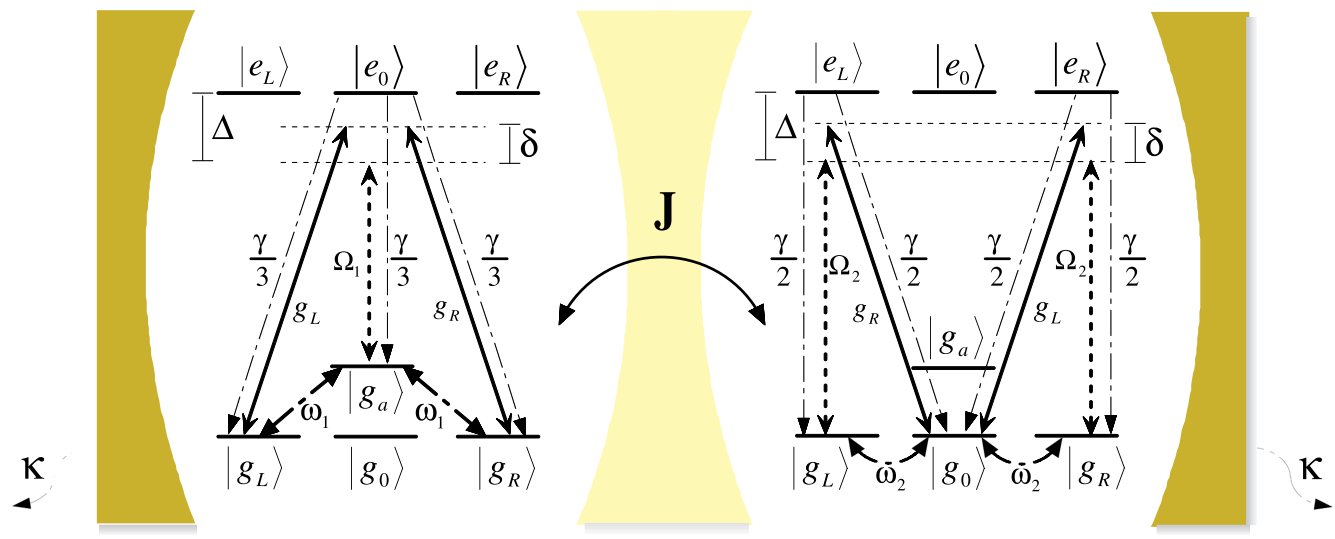

Figure $1 \mid$ Setup for dissipative preparation of three-dimensional entangled state between two ${ }^{87} \mathrm{Rb}$ atoms trapped in two bimode coupled cavities. States $\left|g_{L}\right\rangle,\left|g_{0}\right\rangle,\left|g_{R}\right\rangle$, and $\left|g_{a}\right\rangle$ correspond to atomic levels $\left|F=1, m_{f}=-1\right\rangle,\left|F=1, m_{f}=0\right\rangle,\left|F=1, m_{f}=1\right\rangle$ and $\left|F=2, m_{f}=0\right\rangle$ of $5 S_{1 / 2}$, respectively. And $\left|e_{L}\right\rangle,\left|e_{0}\right\rangle$ and $\left|e_{R}\right\rangle$ correspond to atomic levels $\left|F=1, m_{f}=-1\right\rangle,\left|F=1, m_{f}=0\right\rangle$ and $\left|F=1, m_{f}=1\right\rangle$ of $5 P_{3 / 2}$, respectively. 
governed by the master equation

$$
\dot{\rho}=-i[H, \rho]+\sum_{j}\left[L^{j} \rho L^{j \dagger}-\frac{1}{2}\left(L^{j \dagger} L^{j} \rho+\rho L^{j \dagger} L^{j}\right)\right] .
$$

Under the condition that the Rabi frequency $\Omega$ of the optical pumping laser is sufficiently weak, the excited states of the atoms and the cavity field modes can be adiabatically eliminated when the excited states are not initially populated. In this case, according to the effective operator method in Ref. 47, we can get the effective master equation as

$\dot{\rho}=-i\left[H_{\mathrm{eff}}, \rho\right]+\sum_{j}\left[L_{\mathrm{eff}}^{j} \rho L_{\mathrm{eff}}^{j \dagger}-\frac{1}{2}\left(L_{\mathrm{eff}}^{j \dagger} L_{\mathrm{eff}}^{j} \rho+\rho L_{\mathrm{eff}}^{j \dagger} L_{\mathrm{eff}}^{j}\right)\right]$,

where

$$
\begin{aligned}
& H_{\mathrm{eff}}=-\frac{1}{2}\left[V_{-} H_{\mathrm{NH}}^{-1} V_{+}+V_{-}\left(H_{\mathrm{NH}}^{-1}\right)^{\dagger} V_{+}\right]+H_{g}, \\
& L_{\mathrm{eff}}^{j}=L^{j} H_{\mathrm{NH}}^{-1} V_{+} .
\end{aligned}
$$

In Eq. (8), $H_{\mathrm{NH}}=H_{0}-\frac{i}{2} \sum_{j} L^{j \dagger} L^{j}$ is a non-Hermitian Hamiltonian, and its inverted matrix is $H_{\mathrm{NH}}^{-1}$.

In the following text, we use the effective operator method to simplify the system and research the dissipative process. Nevertheless, for the sake of preciseness, full hamiltonian $H$ rather than $H_{\text {eff }}$ is used for numerical simulation to assess the performance of this scheme.

Use spontaneous emission as resource. In this subsection, aiming to gain better insight into the effect of spontaneous emission on the preparation of a three-dimensional entanglement, we first consider a perfect cavity without decay. According to Eq. (8), we have the effective Hamiltonian

$$
\begin{aligned}
& H_{\mathrm{eff}}=\Omega^{2} \operatorname{Re}\left[\frac{-\widetilde{J^{2}}}{g^{2} \delta+\widetilde{J^{2}} \widetilde{\Delta}}\right]\left(\left|g_{L} g_{L}\right\rangle\left\langle g_{L} g_{L}|+| g_{R} g_{R}\right\rangle\left\langle g_{R} g_{R}|+| T_{3}\right\rangle\left\langle T_{3}\right|\right) \\
& +\Omega^{2} \operatorname{Re}\left[\frac{-\widetilde{J^{2}}}{2 g^{2} \delta+\widetilde{J^{2}} \widetilde{\Delta}}+\frac{-\widetilde{J^{2}}}{g^{2} \delta+\widetilde{J^{2}} \widetilde{\Delta}}\right]\left(\left|g_{a} g_{L}\right\rangle\left\langle g_{a} g_{L}|+| g_{a} g_{R}\right\rangle\left\langle g_{a} g_{R}\right|\right) \\
& +\frac{\Omega^{2}}{3} \operatorname{Re}\left[\frac{g^{2}(4 J+5 \delta)+3 \widetilde{J^{2}} \widetilde{\Delta}}{2 g^{4}-3 g^{2} \delta \widetilde{\Delta}-\widetilde{J}^{2} \widetilde{\Delta}^{2}}\right]\left|T_{1}\right\rangle\left\langle T_{1}\right| \\
& +\frac{\Omega^{2}}{3} \operatorname{Re}\left[\frac{-4 g^{2}(J-\delta)+3 \widetilde{J^{2}} \widetilde{\Delta}}{2 g^{4}-3 g^{2} \delta \widetilde{\Delta}-\widetilde{J}^{2} \widetilde{\Delta}^{2}}\right]\left|T_{2}\right\rangle\left\langle T_{2}\right| \\
& +\frac{\sqrt{2} \Omega^{2}}{3} \operatorname{Re}\left[\frac{-g^{2}(J-\delta)}{2 g^{4}-3 g^{2} \delta \widetilde{\Delta}-\widetilde{J}^{2} \widetilde{\Delta}^{2}}\right]\left(\left|T_{1}\right\rangle\left\langle T_{2}|+| T_{2}\right\rangle\left\langle T_{1}\right|\right)+H_{g},
\end{aligned}
$$

in which

$$
\begin{aligned}
\left|T_{1}\right\rangle & =\frac{1}{\sqrt{3}}\left(\left|g_{L} g_{R}\right\rangle+\left|g_{R} g_{L}\right\rangle+\left|g_{a} g_{0}\right\rangle\right), \\
\left|T_{2}\right\rangle & =\frac{1}{\sqrt{6}}\left(\left|g_{L} g_{R}\right\rangle+\left|g_{R} g_{L}\right\rangle-2\left|g_{a} g_{0}\right\rangle\right), \\
\left|T_{3}\right\rangle & =\frac{1}{\sqrt{2}}\left(\left|g_{L} g_{R}\right\rangle-\left|g_{R} g_{L}\right\rangle\right), \\
\widetilde{J^{2}} & =J^{2}-\delta^{2} \\
\widetilde{\Delta} & =\Delta-\frac{i \gamma}{2} .
\end{aligned}
$$

And $\left|T_{1}\right\rangle$ is the desired three-dimensional entangled state. In order to understand the roles of $H_{g}$ more clearly, we rewrite it as

$$
\begin{aligned}
H_{g} & =\frac{\sqrt{6} \omega}{2}\left(\left|g_{a} g_{L}\right\rangle+\left|g_{a} g_{R}\right\rangle-\left|g_{L} g_{0}\right\rangle-\left|g_{R} g_{0}\right\rangle\right)\left\langle T_{2}\right| \\
& +\frac{\sqrt{2} \omega}{2}\left(\left|g_{a} g_{R}\right\rangle+\left|g_{R} g_{0}\right\rangle-\left|g_{a} g_{L}\right\rangle-\left|g_{L} g_{0}\right\rangle\right)\left\langle T_{3}\right| \\
& +\omega\left(\left|g_{a} g_{L}\right\rangle-\left|g_{L} g_{0}\right\rangle\right)\left\langleg _ { L } g _ { L } \left|+\omega\left(\left|g_{a} g_{R}\right\rangle-\left|g_{R} g_{0}\right\rangle\right)\left\langle g_{R} g_{R}\right|+\right.\right.\text { H.c.. }
\end{aligned}
$$

In addition, on the basis of Eq. (8), the effective Lindblad operators induced by spontaneous emission are

$$
\begin{aligned}
L_{\mathrm{eff}}^{\gamma_{1}, g_{L(a, R)}}= & \sqrt{\frac{\gamma}{3}} \frac{\Omega \widetilde{J^{2}}}{2 g^{2} \delta+\widetilde{J^{2}} \widetilde{\Delta}}\left(\left|g_{L(a, R)} g_{L}\right\rangle\left\langle g_{a} g_{L}|+| g_{L(a, R)} g_{R}\right\rangle\left\langle g_{a} g_{R}\right|\right) \\
& +\frac{\sqrt{\gamma} \Omega}{3}\left[\frac{-g^{2}(2 J+\delta)-\widetilde{J^{2}} \widetilde{\Delta}}{2 g^{4}-3 g^{2} \delta \widetilde{\Delta}-\widetilde{J}^{2} \widetilde{\Delta}^{2}}\right]\left|g_{L(a, R)} g_{0}\right\rangle\left\langle T_{1}\right| \\
& +\frac{\sqrt{2 \gamma} \Omega}{3}\left[\frac{-g^{2}(J-\delta)+\widetilde{J^{2}} \widetilde{\Delta}}{2 g^{4}-3 g^{2} \delta \widetilde{\Delta}-\widetilde{J}^{2} \widetilde{\Delta^{2}}}\right]\left|g_{L(a, R)} g_{0}\right\rangle\left\langle T_{2}\right|,
\end{aligned}
$$

$$
\begin{aligned}
L_{\mathrm{eff}}^{\gamma_{2}, g_{L(R)}} & =\frac{\sqrt{6 \gamma} \Omega}{6}\left[\frac{-g^{2}(J+2 \delta)-\widetilde{J^{2}} \widetilde{\Delta}}{2 g^{4}-3 g^{2} \delta \widetilde{\Delta}-\widetilde{J}^{2} \widetilde{\Delta}^{2}}\right]\left|g_{R(L)} g_{L(R)}\right\rangle\left\langle T_{1}\right| \\
& +\frac{\sqrt{3 \gamma} \Omega}{6}\left[\frac{2 g^{2}(J-\delta)-\widetilde{J^{2}} \widetilde{\Delta}}{2 g^{4}-3 g^{2} \delta \widetilde{\Delta}-\widetilde{J}^{2} \widetilde{\Delta}^{2}}\right]\left|g_{R(L)} g_{L(R)}\right\rangle\left\langle T_{2}\right| \\
& +\frac{\sqrt{2 \gamma} \Omega}{2} \frac{\widetilde{J^{2}}}{g^{2} \delta+\widetilde{J^{2}} \widetilde{\Delta}}\left(\left|g_{L(R)} g_{L(R)}\right\rangle\left\langle g_{L(R)} g_{L(R)}|+| g_{a} g_{L(R)}\right\rangle\left\langle g_{a} g_{L(R)}\right|\right. \\
& \left.-(+) \frac{1}{\sqrt{2}}\left|g_{R(L)} g_{L(R)}\right\rangle\left\langle T_{3}\right|\right),
\end{aligned}
$$$$
L_{\text {eff }}^{\gamma_{2}, g_{0}}=L_{\text {eff }}^{\gamma_{2}, g_{0 L}}+L_{\text {eff }}^{\gamma_{2}, g_{0 R}}
$$$$
=\frac{\sqrt{2 \gamma} \Omega}{2} \frac{\widetilde{J^{2}}}{g^{2} \delta+\widetilde{J^{2}} \widetilde{\Delta}}\left[\left|g_{L} g_{0}\right\rangle\left\langle g_{L} g_{L}|+| g_{a} g_{0}\right\rangle\left\langle g_{a} g_{L}|+| g_{a} g_{0}\right\rangle\left\langle g_{a} g_{R}|+| g_{R} g_{0}\right\rangle\left\langle g_{R} g_{R}\right|\right.
$$$$
\left.+\frac{1}{\sqrt{2}}\left(\left|g_{L} g_{0}\right\rangle-\left|g_{R} g_{0}\right\rangle\right)\left\langle T_{3}\right|\right]+\frac{\sqrt{6 \gamma} \Omega}{6}\left[\frac{-g^{2}(J+2 \delta)-\tilde{J}^{2} \widetilde{\Delta}}{2 g^{4}-3 g^{2} \delta \widetilde{\Delta}-\widetilde{J}^{2} \widetilde{\Delta}^{2}}\right]\left(\left|g_{L} g_{0}\right\rangle+\left|g_{R} g_{0}\right\rangle\right)\left\langle T_{1}\right|
$$$$
+\frac{\sqrt{3 \gamma} \Omega}{6}\left[\frac{2 g^{2}(J-\delta)-\widetilde{J^{2}} \widetilde{\Delta}}{2 g^{4}-3 g^{2} \delta \widetilde{\Delta}-\widetilde{J}^{2} \widetilde{\Delta}^{2}}\right]\left(\left|g_{L} g_{0}\right\rangle+\left|g_{R} g_{0}\right\rangle\right)\left\langle T_{2}\right|
$$

It is important to note that if $\Delta \gg \gamma, J \geq 2 g / 3$, and the cavity detuning from two photon resonance $\delta$ satisfies the condition $\delta=\left(g^{2}+\sqrt{g^{4}+4 J^{2} \Delta^{2}}\right) /(2 \Delta)$, other effective decay channels are approximately ignored except the following dominant parts

$$
\begin{aligned}
L_{\mathrm{eff}}^{\gamma_{2}, g_{L(R)}}= & \frac{\sqrt{2 \gamma} \Omega}{2} \frac{\widetilde{J^{2}}}{g^{2} \delta+\widetilde{J^{2}} \widetilde{\Delta}}\left(\left|g_{L(R)} g_{L(R)}\right\rangle\left\langle g_{L(R)} g_{L(R)}|+| g_{a} g_{L(R)}\right\rangle\left\langle g_{a} g_{L(R)}\right|\right. \\
& \left.-(+) \frac{1}{\sqrt{2}}\left|g_{R(L)} g_{L(R)}\right\rangle\left\langle T_{3}\right|\right), \\
L_{\mathrm{eff}}^{\gamma_{2}, g_{0}}= & \frac{\sqrt{2 \gamma} \Omega}{2} \frac{\widetilde{J^{2}}}{g^{2} \delta+\widetilde{J^{2}} \widetilde{\Delta}} \\
& \times\left[\left|g_{L} g_{0}\right\rangle\left\langle g_{L} g_{L}|+| g_{a} g_{0}\right\rangle\left\langle g_{a} g_{L}|+| g_{a} g_{0}\right\rangle\left\langle g_{a} g_{R}|+| g_{R} g_{0}\right\rangle\left\langle g_{R} g_{R}\right|\right. \\
& \left.+\frac{1}{\sqrt{2}}\left(\left|g_{L} g_{0}\right\rangle-\left|g_{R} g_{0}\right\rangle\right)\left\langle T_{3}\right|\right] .
\end{aligned}
$$

Since $\left|g_{L} g_{R}\right\rangle,\left|g_{R} g_{L}\right\rangle$ and $\left|g_{a} g_{0}\right\rangle$ can be represented by $\left|T_{1}\right\rangle,\left|T_{2}\right\rangle$ and $\left|T_{3}\right\rangle$, the dissipative dynamics in Eq. (13) would transfer any initial states into the subspace composed of $\left|T_{1}\right\rangle,\left|T_{2}\right\rangle,\left|g_{L} g_{0}\right\rangle$ and $\left|g_{R} g_{0}\right\rangle$. 
Besides, coherent dynamics governed by Eq. (9) can be decomposed into two parts, the terms consisting of $\Omega^{2}$ (we use $O\left(\Omega^{2}\right)$ to denote them) and $H_{g} . O\left(\Omega^{2}\right)$ make no contribution to state transition except the term, $\left(\sqrt{2} \Omega^{2} / 3\right) * \operatorname{Re}\left[-g^{2}(J-\delta) /\left(2 g^{4}-3 g^{2} \delta \widetilde{\Delta}-\widetilde{J^{2}} \widetilde{\Delta}^{2}\right)\right]$ $\left(\left|T_{1}\right\rangle\left\langle T_{2}|+| T_{2}\right\rangle\left\langle T_{1}\right|\right)$, which induces the transition $\left|T_{1}\right\rangle \leftrightarrow\left|T_{2}\right\rangle$. From Eq. (11), one can see that $H_{g}$ keeps $\left|T_{1}\right\rangle$ invariant while makes $\left|T_{2}\right\rangle, \quad\left|g_{L} g_{0}\right\rangle$ and $\left|g_{R} g_{0}\right\rangle$ evolve out of the subspace. If $\left(\sqrt{2} \Omega^{2} / 3\right) * \operatorname{Re}\left[-g^{2}(J-\delta) /\left(2 g^{4}-3 g^{2} \delta \widetilde{\Delta}-\widetilde{J}^{2} \widetilde{\Delta}^{2}\right)\right] \ll\{\sqrt{6} \omega / 2$, $\sqrt{2} \omega / 2, \omega\}$ is satisfied, the coherent dynamics contributed to the state transitions is mainly governed by $H_{g}$ rather than $O\left(\Omega^{2}\right)$. And the condition $\omega_{1}=-\omega_{2}$ is critical since it guarantees $\left|T_{1}\right\rangle$ to be the dark state of $H_{g}$. In Fig. 2, we plot the fidelity of state $\left|T_{1}\right\rangle, F=$ $\left\langle T_{1}|\rho| T_{1}\right\rangle$, with the above conditions been satisfied. From Fig. 2 , one can see that the desired state can be achieved with a high fidelity.

Use cavity decay as resource. In this subsection, aiming to gain better insight into the effect of cavity decay on the preparation of a three-dimensional entanglement, we do not consider spontaneous emission here.

(a)

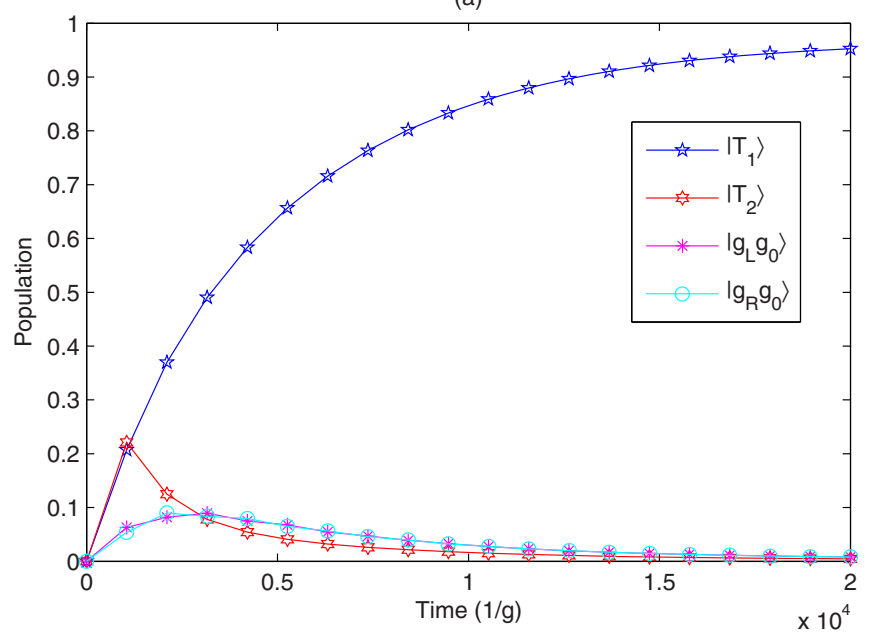

(b)

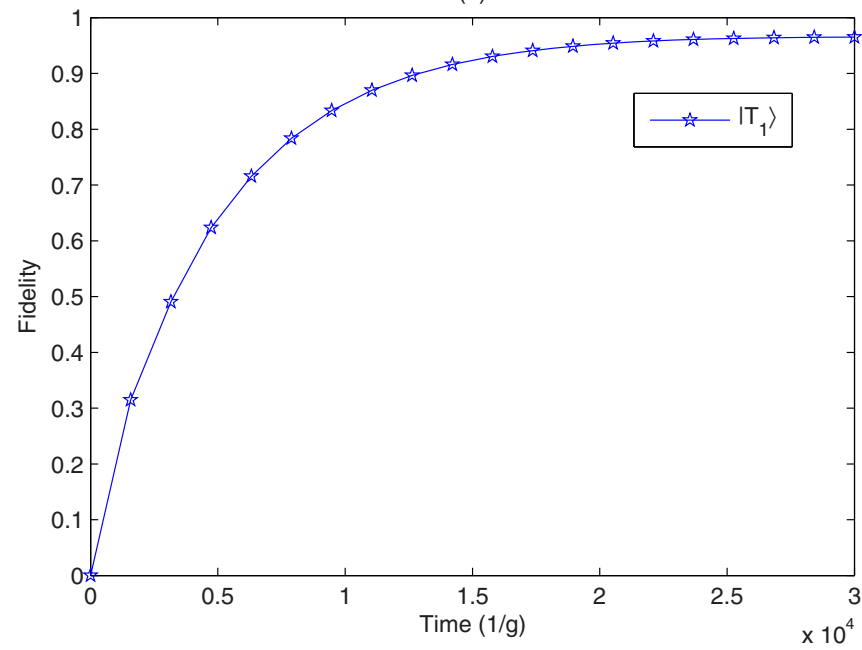

Figure $2 \mid$ (a) Population for the states in the steady subspace of the spontaneous-emission-based case from an initial state $\left|g_{a} g_{L}\right\rangle$. (b) Fidelity of $\left|T_{1}\right\rangle$ state from an initial state $\left|g_{a} g_{L}\right\rangle$. Both figures are plotted under the given parameters $\Omega=0.01 g, \omega=0.2 \Omega, \omega=0.04 g, \kappa=0, \Delta=g, J=6 g$.
According to Eq. (8), the effective Hamiltonian is achieved as

$$
\begin{aligned}
H_{\mathrm{eff}}= & \Omega^{2} \operatorname{Re}\left[\frac{-\widetilde{J^{2}}}{g^{2} \widetilde{\delta}+\Delta \widetilde{J^{\prime}}}\right]\left(\left|g_{L} g_{L}\right\rangle\left\langle g_{L} g_{L}|+| g_{R} g_{R}\right\rangle\left\langle g_{R} g_{R}|+| T_{3}\right\rangle\left\langle T_{3}\right|\right) \\
& +\Omega^{2} \operatorname{Re}\left[\frac{-\widetilde{J^{2}}}{g^{2} \widetilde{\delta}+\Delta{\widetilde{J^{\prime}}}^{\prime}}+\frac{-\widetilde{J^{\prime}}}{2{g^{2}}^{2}+\Delta \widetilde{J^{\prime}}}\right]\left(\left|g_{a} g_{L}\right\rangle\left\langle g_{a} g_{L}|+| g_{a} g_{R}\right\rangle\left\langle g_{a} g_{R}\right|\right) \\
& +\frac{\Omega^{2}}{3} \operatorname{Re}\left[\frac{g^{2}(4 J+5 \widetilde{\delta})+3 \widetilde{J^{\prime}} \Delta}{2 g^{4}-3 g^{2} \widetilde{\delta} \Delta-\widetilde{J^{2}} \Delta^{2}}\right]\left|T_{1}\right\rangle\left\langle T_{1}\right| \\
& +\frac{\Omega^{2}}{3} \operatorname{Re}\left[\frac{-4 g^{2}(J-\widetilde{\delta})+3 \widetilde{J^{\prime}} \Delta}{2 g^{4}-3 g^{2} \widetilde{\delta} \Delta-\widetilde{J^{2}} \Delta^{2}}\right]\left|T_{2}\right\rangle\left\langle T_{2}\right| \\
& +\frac{\sqrt{2} \Omega^{2}}{3}\left[\frac{-g^{2}(J-\widetilde{\delta})}{2 g^{4}-3 g^{2} \widetilde{\delta} \Delta-\widetilde{J^{2}} \Delta^{2}}\right]\left(\left|T_{1}\right\rangle\left\langle T_{2}|+| T_{2}\right\rangle\left\langle T_{1}\right|\right)+H_{g},
\end{aligned}
$$

in which

$$
\begin{aligned}
\widetilde{J}^{\prime} & =J^{2}-(\widetilde{\delta})^{2}, \\
\widetilde{\delta} & =\delta-\frac{i k}{2} .
\end{aligned}
$$

Besides, the effective Lindblad operators induced by cavity decay can be written as

$$
\begin{aligned}
& L_{\text {eff }}^{\kappa, c_{L 1(R 1)}}=\frac{\sqrt{2 \kappa} \Omega}{2} \frac{g(J+\widetilde{\delta})}{2 g^{2} \delta+\widetilde{\Delta J^{2}}}\left(\left|g_{L(R)} g_{L}\right\rangle\left\langle g_{a} g_{L}|+| g_{R(L)} g_{R}\right\rangle\left\langle g_{a} g_{R}\right|\right) \\
& -\frac{\sqrt{2 \kappa} \Omega}{2} \frac{g(J+\widetilde{\delta})}{g^{2} \delta+\Delta \widetilde{J}^{\prime}} \\
& \times\left(\left|g_{a} g_{0}\right\rangle\left\langle g_{a} g_{R(L)}|+| g_{R(L)} g_{0}\right\rangle\left\langle g_{R(L)} g_{R(L)}\left|+(-) \frac{1}{\sqrt{2}}\right| g_{L(R)} g_{0}\right\rangle\left\langle T_{3}\right|\right) \\
& -\frac{\sqrt{6 \kappa} \Omega}{6} \frac{g^{3}}{2 g^{4}-3 g^{2} \widetilde{\delta} \Delta-\widetilde{J}^{\prime} \Delta^{2}}\left|g_{L(R)} g_{0}\right\rangle\left\langle T_{1}\right| \\
& +\frac{\sqrt{3 \kappa} \Omega}{6} \frac{g\left(-4 g^{2}+3 \Delta(J+\widetilde{\delta})\right)}{2 g^{4}-3 g^{2} \widetilde{\delta} \Delta-\widetilde{J}^{\prime} \Delta^{2}}\left|g_{L(R)} g_{0}\right\rangle\left\langle T_{2}\right|, \\
& L_{\mathrm{eff}}^{\kappa, c_{L 2(R 2)}}=-\frac{\sqrt{2 \kappa} \Omega}{2} \frac{g(J-\widetilde{\delta})}{2 g^{2} \widetilde{\delta}+\Delta{\widetilde{J^{2}}}^{\prime}}\left(\left|g_{L(R)} g_{L}\right\rangle\left\langle g_{a} g_{L}|+| g_{L(R)} g_{R}\right\rangle\left\langle g_{a} g_{R}\right|\right) \\
& -\frac{\sqrt{2 \kappa} \Omega}{2} \frac{g(J-\widetilde{\delta})}{g^{2} \widetilde{\delta}+\Delta{\widetilde{J^{\prime}}}^{\prime}} \\
& \times\left(\left|g_{a} g_{0}\right\rangle\left\langle g_{a} g_{R(L)}|+| g_{R(L)} g_{0}\right\rangle\left\langle g_{R(L)} g_{R(L)}\left|+(-) \frac{1}{\sqrt{2}}\right| g_{L(R)} g_{0}\right\rangle\left\langle T_{3}\right|\right) \\
& +\frac{\sqrt{6 \kappa} \Omega}{6} \frac{g\left(3 g^{2}+2 \Delta(J-\widetilde{\delta})\right)}{2 g^{4}-3 g^{2} \widetilde{\delta} \Delta-\widetilde{J}^{\prime} \Delta^{2}}\left|g_{L(R)} g_{0}\right\rangle\left\langle T_{1}\right| \\
& -\frac{\sqrt{3 \kappa} \Omega}{6} \frac{g \Delta(J-\widetilde{\delta})}{2 g^{4}-3 g^{2} \widetilde{\delta} \Delta-\widetilde{J}^{\prime} \Delta^{2}}\left|g_{L(R)} g_{0}\right\rangle\left\langle T_{2}\right| \text {. }
\end{aligned}
$$

It is noticeable if $\delta \gg \kappa, J \geq 2 g / 3$, and the cavity detuning from two photon resonance $\delta$ meets the condition 
$\delta=\left(g^{2}+\sqrt{g^{4}+4 J^{2} \Delta^{2}}\right) /(2 \Delta)$, other effective decay channels are approximately ignored except the following dominant terms

$$
\begin{aligned}
& L_{\text {eff }}^{\kappa, c_{L 1(R 1)}}=-\frac{\sqrt{2 \kappa} \Omega}{2} \frac{g(J+\widetilde{\delta})}{g^{2} \delta+\Delta{\widetilde{J^{2}}}^{\prime}} \\
& \times\left(\left|g_{a} g_{0}\right\rangle\left\langle g_{a} g_{R(L)}|+| g_{R(L)} g_{0}\right\rangle\left\langle g_{R(L)} g_{R(L)}\left|+(-) \frac{1}{\sqrt{2}}\right| g_{L(R)} g_{0}\right\rangle\left\langle T_{3}\right|\right), \\
& L_{\mathrm{eff}}^{\kappa, C_{L 2(R 2)}}=-\frac{\sqrt{2 \kappa} \Omega}{2} \frac{g(J-\widetilde{\delta})}{g^{2} \widetilde{\delta}+\Delta \widetilde{J^{2}}}\left(\left|g_{a} g_{0}\right\rangle\left\langle g_{a} g_{R(L)}|+| g_{R(L)} g_{0}\right\rangle\left\langle g_{R(L)} g_{R(L)}\right|\right. \\
& \left.+(-) \frac{1}{\sqrt{2}}\left|g_{L(R)} g_{0}\right\rangle\left\langle T_{3}\right|\right) .
\end{aligned}
$$

Since $\left|g_{L} g_{R}\right\rangle,\left|g_{R} g_{L}\right\rangle$ and $\left|g_{a} g_{0}\right\rangle$ can be represented by $\left|T_{1}\right\rangle,\left|T_{2}\right\rangle$ and $\left|T_{3}\right\rangle$, the dissipative channels in Eq. (17) would translate any initial states into the subspace composed of $\left|T_{1}\right\rangle,\left|T_{2}\right\rangle,\left|g_{L} g_{0}\right\rangle$ and $\left|g_{R} g_{0}\right\rangle$. Similar to Eq. (9), the coherent dynamics governed by Eq. (14) could also be divided into two parts, $O^{\prime}\left(\Omega^{2}\right)$ and $H_{g} . O^{\prime}\left(\Omega^{2}\right)$ make no contribution to state transitions except the term, $\left(\sqrt{2} \Omega^{2} / 3\right) * \operatorname{Re}$ $\left[-g^{2}(J-\widetilde{\delta}) /\left(2 g^{4}-3 g^{2} \widetilde{\delta} \Delta-\widetilde{J}^{\prime} \Delta^{2}\right)\right]\left(\left|T_{1}\right\rangle\left\langle T_{2}|+| T_{2}\right\rangle\left\langle T_{1}\right|\right)$. Thus, if $\left(\sqrt{2} \Omega^{2} / 3\right) * \operatorname{Re}\left[-g^{2}(J-\widetilde{\delta}) /\left(2 g^{4}-3 g^{2} \widetilde{\delta} \Delta-\widetilde{J}^{2} \Delta^{2}\right)\right] \ll$ $\{\sqrt{6} \omega / 2, \sqrt{2} \omega / 2, \omega\}$ is satisfied, the unitary dynamics contributed to the state transitions is mainly governed by $H_{g}$ rather than $O^{\prime}\left(\Omega^{2}\right)$. From Eq. (11), one can see that $H_{g}$ guarantee $\left|T_{1}\right\rangle$ being invariant while the other three states being driven out of the steady subspace. Therefore, the population of the desired state increases over time for any initial state. We plot the population and fidelity in Fig. 3 under one group of the optimal parameters that satisfy the above conditions. Nevertheless, the results do about $20 \%$ worse than that of the spontaneous-emission-based case because states $\left|g_{L} g_{0}\right\rangle$ and $\left|g_{R} g_{0}\right\rangle$ both occupy a population more than $10 \%$ when the system approaches to stabilization. This situation is not hard to understand through comparing the dominant dissipative channels of the spontaneous-emission-based case in Eq. (13) with that of the cavitydecay-based case in Eq. (17). For the sake of analysis, we plot the transitions of the dressed states induced by Eq. (11), (13) and (17) in Fig. 4(a), 4(b) and 4(c), respectively. Compared with Fig. 4(b), Fig. 4(c) has two more channels directed to $\left|g_{L} g_{0}\right\rangle$ and two more channels directed to $\left|g_{R} g_{0}\right\rangle$. Under the control of the same Hamiltonian $H_{g}$, the difference of the dominant dissipative channels mentioned above leads to the result that the performance of the cavity-decay-based case is lower than that of the spontaneous-emission-based case.

In this paragraph, we aim to use the feedback control ${ }^{54-63}$ to improve the performance of the cavity-decay-based case. The idea of the feedback control is depicted in Fig 5: the cavity decay is measured by a detector $D$ whose signal triggers a feedback laser pulse with evolution operator $\hat{U}$. The dynamics include feedback control is governed by the master equation

$$
\dot{\rho}=-i[H, \rho]+\kappa \sum_{\ell}\left[\hat{U}_{\ell} \hat{\ell} \rho \hat{\ell}^{\dagger} \hat{U}_{\ell}^{\dagger}-\frac{1}{2}\left(\hat{\ell}^{\dagger} \hat{\ell} \rho+\rho \hat{\ell}^{\dagger} \hat{\ell}\right)\right],
$$

in which $\ell$ denotes $c_{L 1}, c_{R 1}, c_{L 2}$ and $c_{R 2}$, respectively. $\hat{U}_{\ell}$ is the feedback operation which could be implemented through choosing suitable feedback laser pulse $\mathrm{e}^{60-62}$. In order to decrease the population of states $\left|g_{L} g_{0}\right\rangle$ and $\left|g_{R} g_{0}\right\rangle$, one can design many different feedback operations. We first choose $\hat{U}_{\mathcal{C}_{R 1}}=\exp \left(i \lambda_{1} \sigma_{x 1}\right)\left(\lambda_{1}\right.$ denotes the feedback strength and $\sigma_{x 1}$ is associated with the feedback pulse Hamiltonian ${ }^{60-62}$ ), which means feedback operation $\hat{U}_{c_{R 1}}$ would be applied on atom 2 when photon in mode $\hat{c}_{R 1}$ is detected, to study the effect of the (a)

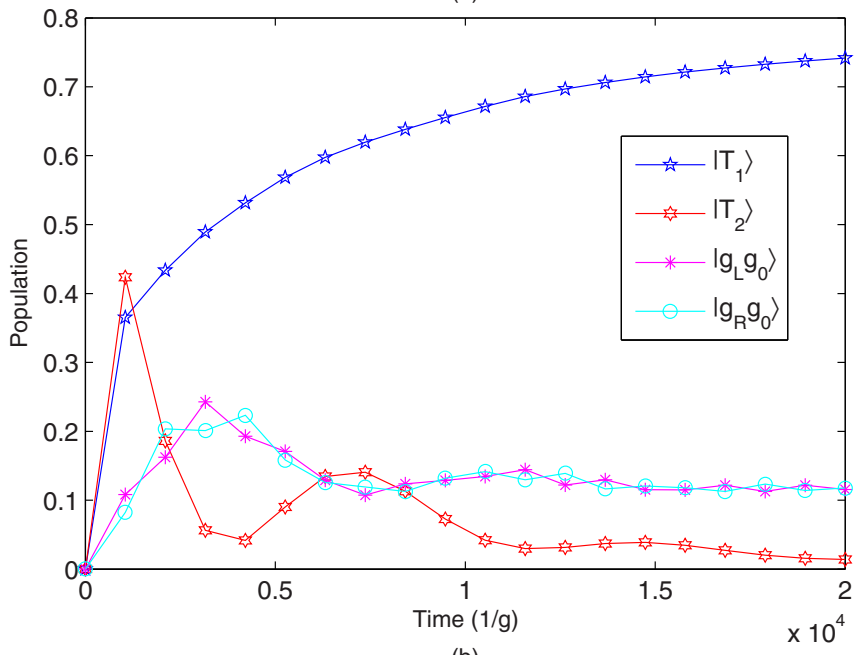

(b)

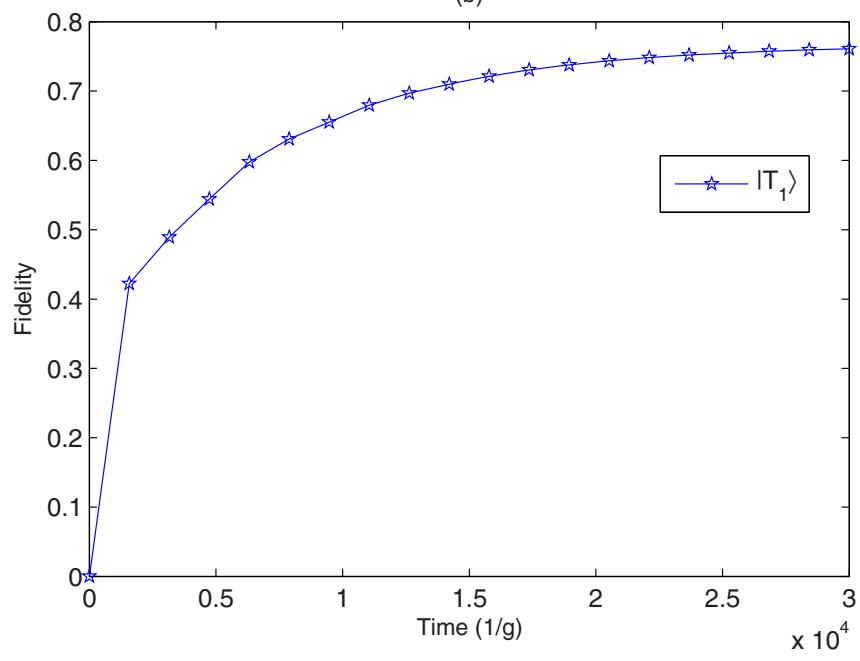

Figure $3 \mid$ (a) Population for the states in the steady subspace of the cavitydecay-based case from an initial state $\left|g_{a} g_{L}\right\rangle$. (b) Fidelity of $\left|T_{1}\right\rangle$ from an initial state $\left|g_{a} g_{L}\right\rangle$. Both figures are plotted under the given parameters $\Omega=0.03 g, \omega=0.05 \Omega, \gamma=0, \kappa=0.05 g, \Delta=g, J=6 g$.

feedback control. The photon in mode $\hat{c}_{R 1}$ measured by the detector may emit through the energy level transition $\left|e_{0}\right\rangle_{1} \rightarrow\left|g_{R}\right\rangle_{1}$ of the atom 1 or from the energy level transition $\left|e_{L}\right\rangle_{2} \rightarrow\left|g_{0}\right\rangle_{2}$ of the atom 2. If it emits from atom 1 , the atom 1 is undoubtedly in state $\left|g_{R}\right\rangle_{1}$. While atom 2 has three possible states, $\left|g_{L}\right\rangle_{2},\left|g_{0}\right\rangle_{2}$ or $\left|g_{R}\right\rangle_{2}$. If it emits from atom 2 , the atom 2 will undoubtedly be in state $\left|g_{0}\right\rangle_{2}$. While atom 1 has three possible states, $\left|g_{L}\right\rangle_{1},\left|g_{a}\right\rangle_{1}$ or $\left|g_{R}\right\rangle_{1}$. In short, if the detector detects the cavity decay in mode $\hat{c}_{R 1}$, the state of the system has five possibilities, $\left|g_{R}\right\rangle\left|g_{L}\right\rangle,\left|g_{R}\right\rangle\left|g_{0}\right\rangle,\left|g_{R}\right\rangle\left|g_{R}\right\rangle,\left|g_{L}\right\rangle\left|g_{0}\right\rangle$ and $\left|g_{a}\right\rangle\left|g_{0}\right\rangle$. However, after unitary dynamics (see Fig 4(a) for clarity) and dominant dissipative channels (see Fig 4(c) for clarity) working, $\left|g_{R}\right\rangle\left|g_{L}\right\rangle,\left|g_{R}\right\rangle\left|g_{R}\right\rangle$ and $\left|g_{a}\right\rangle\left|g_{0}\right\rangle$ would be finally transformed to $\left|T_{1}\right\rangle,\left|g_{R}\right\rangle\left|g_{0}\right\rangle$ and $\left|g_{L}\right\rangle\left|g_{0}\right\rangle$ (see Fig. 3(a) for clarity). Thus, if the detector clicks, the possible states of the system which need to be modified are $\left|g_{R}\right\rangle\left|g_{0}\right\rangle$ and $\left|g_{L}\right\rangle\left|g_{0}\right\rangle$. Then, if we choose $\lambda_{1}=\pi / 2$ and $\sigma_{x 1}=\left|g_{0}\right\rangle_{22}\left\langle g_{R}\right|+$ $\left|g_{R}\right\rangle_{22}\left\langle g_{0}\right|, \hat{U}_{c_{R 1}}$ would transform $\left|g_{R}\right\rangle\left|g_{0}\right\rangle$ and $\left|g_{L}\right\rangle\left|g_{0}\right\rangle$ to $\left|g_{R}\right\rangle\left|g_{R}\right\rangle$ and $\left|g_{L}\right\rangle\left|g_{R}\right\rangle$, respectively. As a result, the population of $\left|g_{R}\right\rangle\left|g_{0}\right\rangle$ and $\left|g_{L}\right\rangle\left|g_{0}\right\rangle$ decreases and that of $\left|T_{1}\right\rangle$ increases (see Fig. 6 for clarity). On the other hand, another feedback operation $\hat{U}_{\mathcal{C}_{R 1}}^{\prime}=\exp \left(i \lambda_{2} \sigma_{x 2}\right)$ with $\lambda_{2}=\pi / 2$ and $\sigma_{x 2}=\left|g_{0}\right\rangle_{22}\left\langle e_{R}|+| e_{R}\right\rangle_{22}\left\langle g_{0}\right|$ was also investigated in Fig. 6. Unlike $\hat{U}_{c_{R 1}}, \hat{U}_{c_{R 1}}^{\prime}$ transform $\left|g_{R}\right\rangle\left|g_{0}\right\rangle$ and $\left|g_{L}\right\rangle\left|g_{0}\right\rangle$ to $\left|g_{R}\right\rangle\left|e_{R}\right\rangle$ and $\left|g_{L}\right\rangle\left|e_{R}\right\rangle$, respectively. Subsequently, if $\left|e_{R}\right\rangle_{2}$ is transformed to 


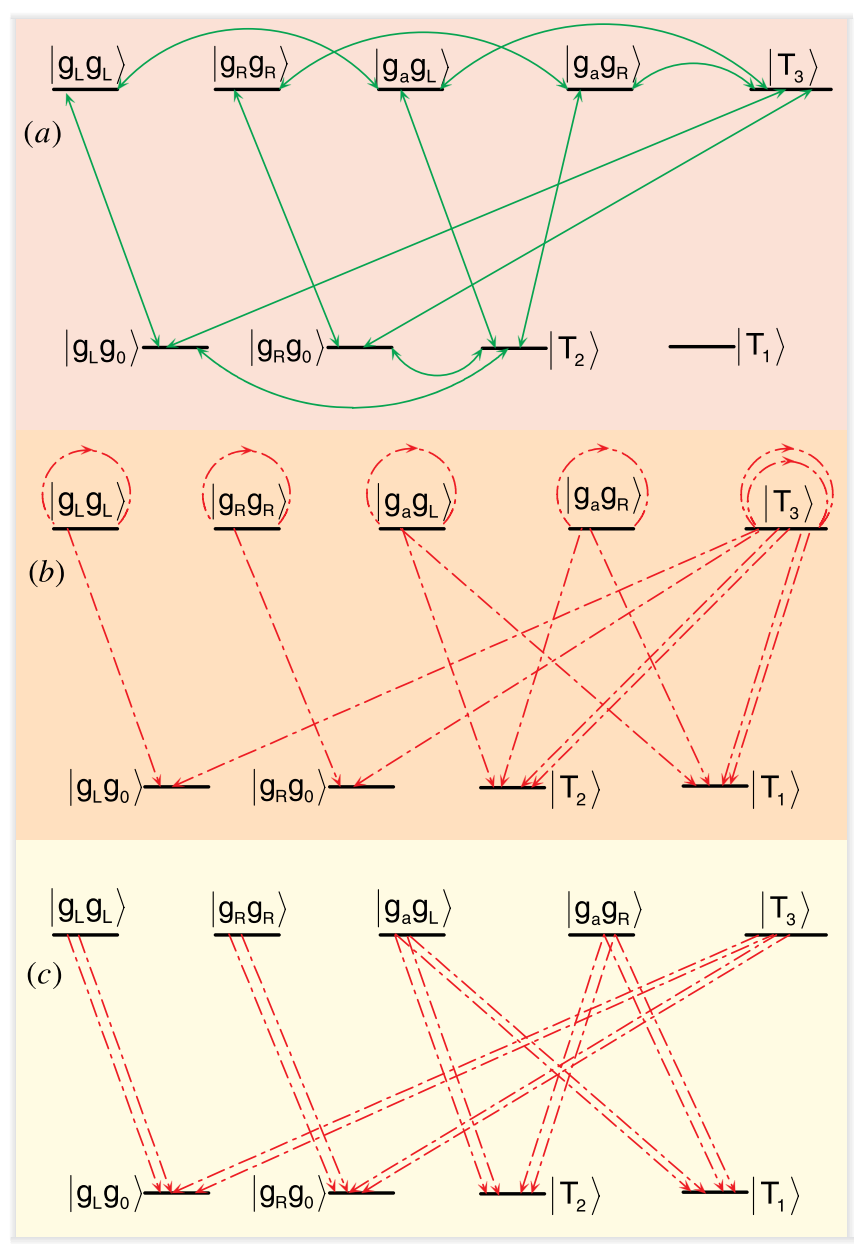

Figure $4 \mid$ (a) Roles of the effective Hamiltonian $H_{g}$, which is drawn according to Eq. (11). (b) Roles of the effective dissipative channels of the spontaneous-emission-based scheme, which is drawn according to Eq. (13). (c) Roles of the effective dissipative channels of the cavity-decaybased scheme, which is drawn according to Eq. (17). It should be noted that $\left|g_{R(L)} g_{L(R)}\right\rangle=\left(\sqrt{2}\left|T_{1}\right\rangle+\left|T_{2}\right\rangle-(+) \sqrt{3}\left|T_{3}\right\rangle\right) / \sqrt{6}$ and $\left|g_{a} g_{0}\right\rangle=\left(\left|T_{1}\right\rangle-\sqrt{2}\left|T_{2}\right\rangle\right) / \sqrt{3}$. And we use $\left|T_{1}\right\rangle,\left|T_{2}\right\rangle$ and $\left|T_{3}\right\rangle$ to replace $\left|g_{R(L)} g_{L(R)}\right\rangle$ and $\left|g_{a} g_{0}\right\rangle$ in Fig. 4(b) and 4(c) for clarity.

$\left|g_{R}\right\rangle_{2}$ through coupling with optical laser $\Omega_{2},\left|g_{R}\right\rangle\left|g_{0}\right\rangle$ and $\left|g_{L}\right\rangle\left|g_{0}\right\rangle$ have been successfully transformed to other states. However, if $\left|e_{R}\right\rangle_{2}$ is transformed to $\left|g_{0}\right\rangle_{2}$ through coupling with cavity mode $a_{L 2}$, our purpose would not be achieved. Therefore, the feedback operation $\hat{U}_{c_{R 1}}^{\prime}$-based case is not as better as $\hat{U}_{c_{R 1}}$-based case.

Simultaneously use both spontaneous emission and cavity decay as resources. Note that the conditions to obtain the dominant decay

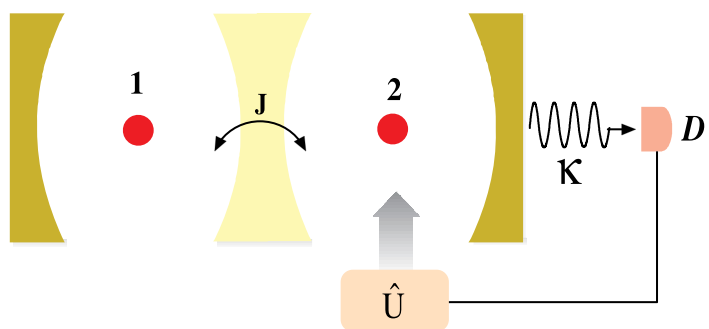

Figure 5 | Schematic view of the quantum feedback control. $D$ is a photon detector. $\hat{U}$ is the feedback operator which could be implemented through well-designed laser field pulse.

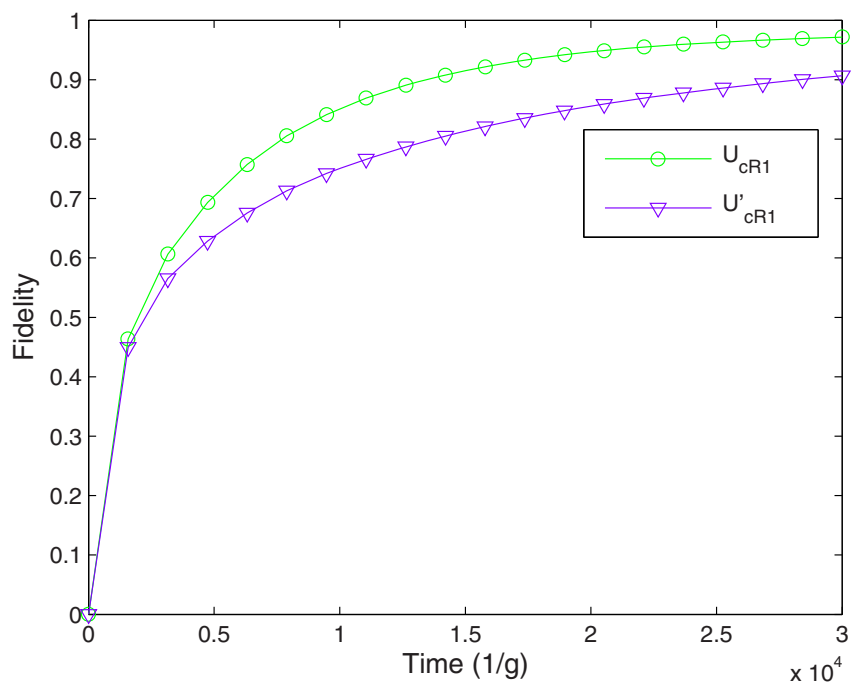

Figure 6 Fidelity of cavity-decay-based case via adding feedback control. The figure is plotted under the parameters $\Omega=0.04 \mathrm{~g}, \omega=0.05 \Omega$, $\gamma=0, \kappa=0.1 \mathrm{~g}, \Delta=g, J=6 \mathrm{~g}$.

channels in Eq. (13) and Eq. (17) can be satisfied at the same time, it is thus possible to use both spontaneous emission and cavity decay to prepare the desired state simultaneously. In Fig. 7, we plot the fidelity of the presented scheme based on spontaneous emission and cavity decay simultaneously, from which we can see that $\left|T_{1}\right\rangle$ can be achieved with the fidelity close to 0.9 under specific parameters. In Fig. 8, we plot the fidelity with parameters the same to Fig. 7 via adding feedback control. Results show that feedback control can improve the fidelity, shorten the time to be steady and improve the robustness on parameters variation.

\section{Discussion}

The main method used here is the effective operator method proposed in Refs. 13, 47. And the main idea of the presented scheme is to leverage dissipative to build effective decay channels and construct the steady state subspace which contains the desired state. Then, effective Hamiltonian is designed to make sure the desired state being its dark state while the others being driven out of the subspace. To see clearly the role of each dissipative factor, we first consider the system without cavity decay, and then consider it without spontaneous emission. Numerical simulation shows that both spontaneous emission and cavity decay could be used as resources for highdimensional entanglement preparation. The only drawback is that cavity-decay-based case is not as ideal as spontaneous-emissionbased case. The difference of the effective dissipative channels between these two cases is the chief cause that gives rise to this phenomenon although the steady state subspaces are the same. Therefore, feedback control is added to improve the performance of the cavity-decay-based case. Interestingly, the conditions to obtain the effective dissipative channels in Eq. (13) and Eq. (17) can be satisfied at the same time, it is thus possible to use both spontaneous emission and cavity decay as resources simultaneously. From Fig. 7, one can see that when $\kappa$ and $\gamma$ are both set to zero, the fidelity is zero. When both dissipative factors increase to $0.01 \mathrm{~g}$, the fidelity will be higher than $90 \%$. This result proves that both $\kappa$ and $\gamma$ are used as resources. However, further increase of the the dissipative factors would decrease the fidelity. This is because $\Delta \gg \gamma$ and $\delta \gg \kappa$, which are the necessary conditions to achieve the dominant dissipative channels in Eq. (13) and Eq. (17) respectively, would not be satisfied well when $\kappa$ and $\gamma$ increase. Therefore, if we calculate the fidelity from a perspective similar to Ref. 22 -that is, suppose $\gamma$ equals to $0.01 \mathrm{~g}$, and $\kappa$ takes several values in a certain range [0.01 g, $0.1 \mathrm{~g}$ ]-then we 


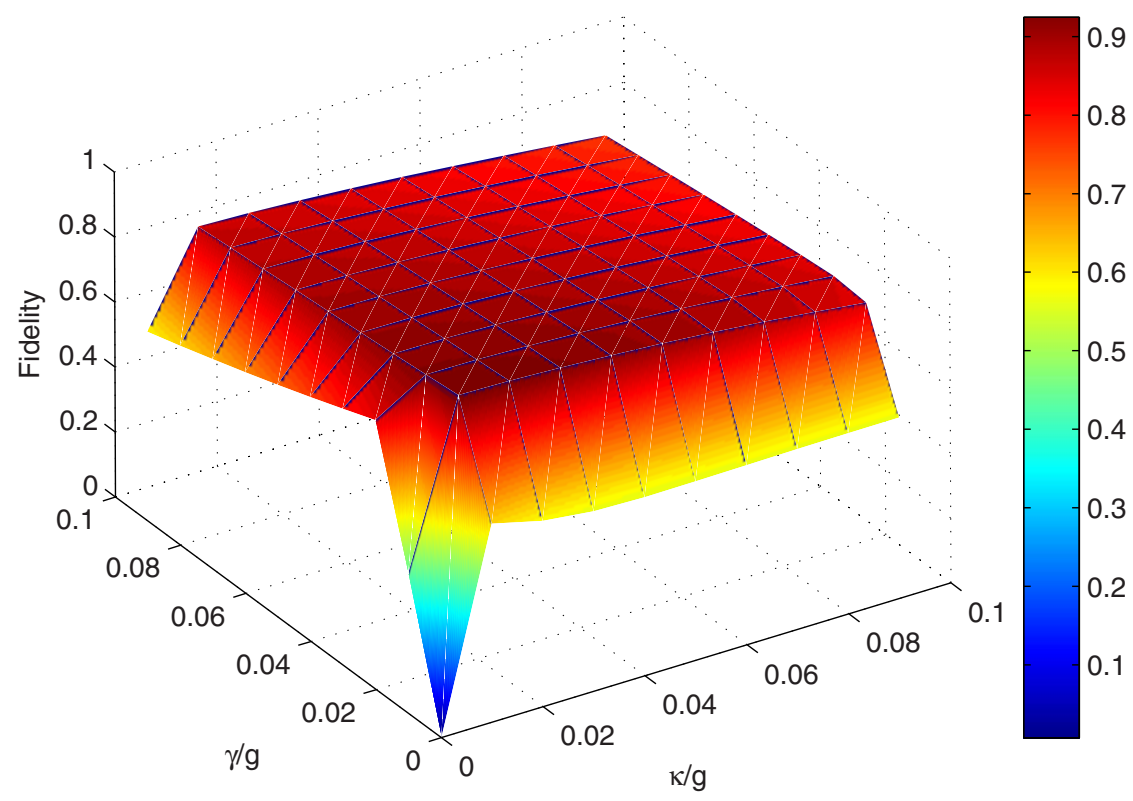

Figure 7 Fidelity of the scheme based on spontaneous emission and cavity decay without feedback control at the time 60000/g. Parameters are chosen as $\Omega=0.03 g, \omega=0.2 \Omega, \Delta=g, J=6 g$. $\kappa$ and $\gamma$ are chosen form 0 to $0.1 \mathrm{~g}$ with regular intervals equal to $0.01 \mathrm{~g}$.

would see that the fidelity decreases as $\kappa$ grows and thus conclude that $\kappa$ plays a negative role for entanglement preparation. Nevertheless, after studying the effect of cavity decay more comprehensively in the present manuscript, we find that it could also be used as resources to prepare entanglement although the performance is not as better as the spontaneous-emission-based case.

For coupled cavity system, coupling strength $g$, cavity decay rate $\kappa$ and the spontaneous emission rate $\gamma$ are stationary. However, we can adjust the parameters $\Omega, \omega, \Delta$ and $\delta$ to achieve the desired state with high fidelity. In the subsection of Basic model, we give two ways to realize the transitions between the degenerate ground states of atom 2. One of them is the degenerate Raman coupling process through using a virtual upper state. The effective Rabi frequency $\omega_{2}$ which can be achieved by this process could be approximately expressed as $\Omega_{1}^{\prime} \Omega_{2}^{\prime}\left[1 /\left(2 \Delta_{1}^{\prime}\right)+1 /\left(2 \Delta_{2}^{\prime}\right)\right]^{64-66}$, where $\Omega_{i}^{\prime}$ denotes the coupling strength between the ground state and the virtual upper state with the corresponding detuning $\Delta_{i}^{\prime}$. That is, in theory, one can achieve the required effective Rabi frequency through choosing the values of $\Omega_{i}^{\prime}$ and $\Delta_{i}^{\prime}$. Without loss of generality, after choosing $\Omega_{1}^{\prime}=\Omega_{2}^{\prime}=0.5 \Omega$ and $\Delta_{1}^{\prime}=\Delta_{2}^{\prime}=5 \Omega$, one can get the effective Rabi frequency $0.05 \Omega$. Even so, in practical case, the effective Rabi frequency $\omega_{2}$ may be smaller than the values used in above numerical simulations. When it happens, one should first adjust $\omega_{1}$ to make it satisfy the condition $\omega_{1}=-\omega_{2}=\omega$. In order to study the effects of $\omega$ decrease, we use another groups of parameters to make numerical simulations in Fig. 9. For the atomic-spontaneous-emission-based case, the parameters in Fig. 2(b) satisfy $\omega=0.2 \Omega=0.002 \mathrm{~g}$, and the time for the system to reach the steady state is about $3 \times 10^{4} / \mathrm{g}$. In contrast, the parameters in Fig. 9(a) satisfy $\omega=0.04 \Omega=0.0002 \mathrm{~g}$, which is smaller than that in Fig. 2(b). And the time for the system to

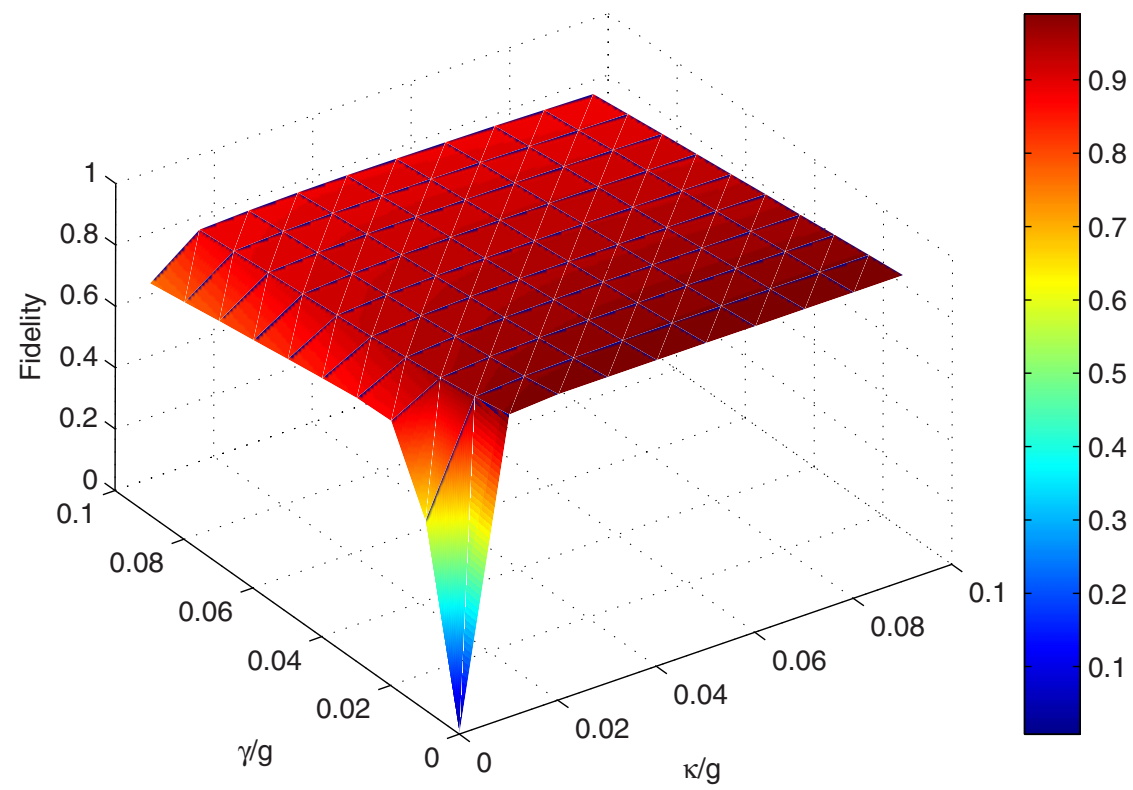

Figure 8 Fidelity of the scheme based on spontaneous emission and cavity decay via adding feedback operations $\hat{U}_{\mathcal{C}_{R 1}}$ at the time $20000 / g$. Parameters chosen here are the same as in Fig. 7. 
(a)

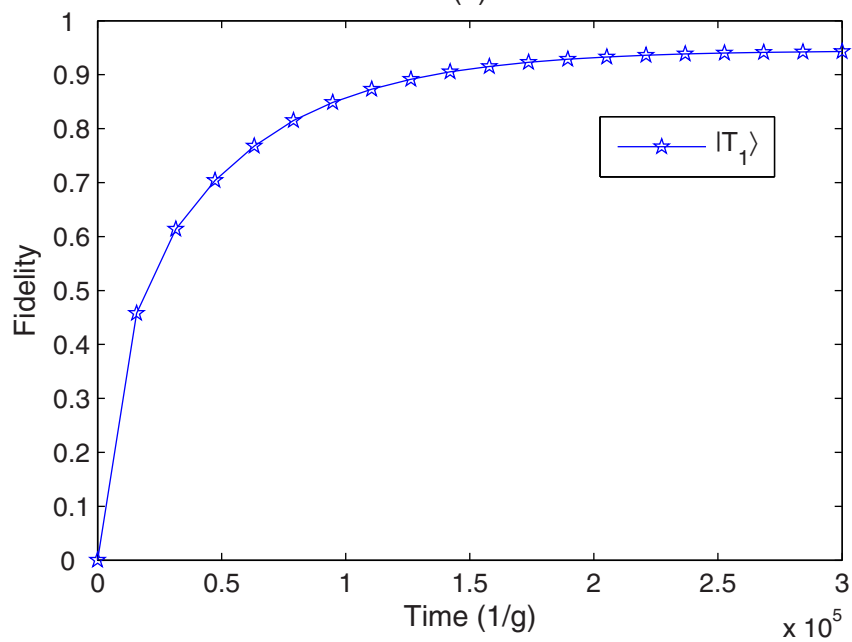

(b)

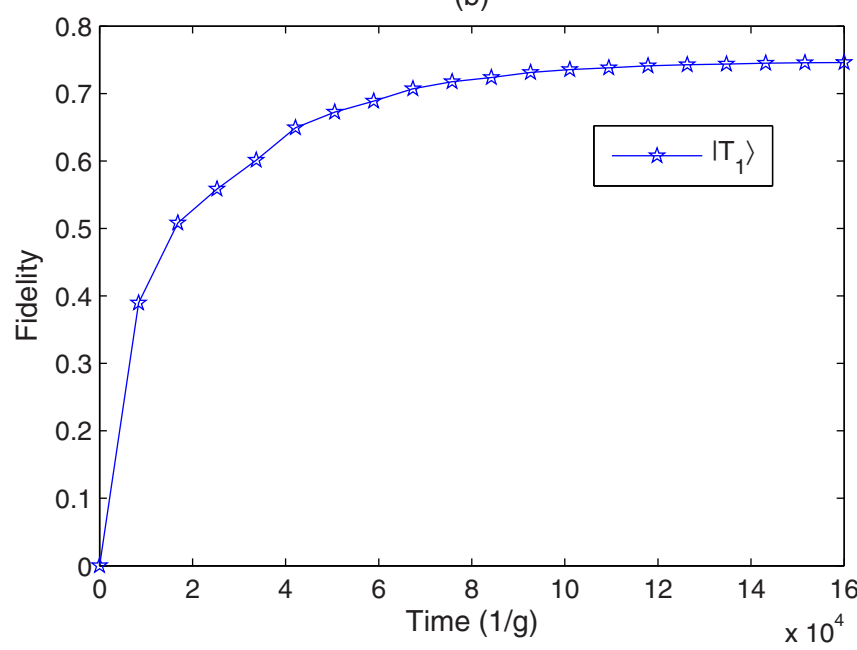

Figure 9 | (a) Fidelity of the spontaneous-emission-based case with $\Omega=$ $0.005 g, \omega=0.04 \Omega$. The rest parameters are the same as in Fig. 2. (b) Fidelity of the cavity-decay-based case with $\Omega=0.007 \mathrm{~g}, \omega=0.021 \Omega$. The rest parameters are the same as in Fig. 3.

reach the stationary state is about $3 \times 10^{5} / g$, which is longer than that in Fig. 2(b). Nevertheless, the final fidelities of the desired steady state in Fig. 2(b) and Fig. 9(a) are more or less the same. For the cavitydecay-based case, the parameters in Fig. 3(b) satisfy $\omega=0.05 \Omega=$ $0.0015 \mathrm{~g}$, and the time for the system to reach the stationary state is about $3 \times 10^{4} / g$. In contrast, the parameters in Fig. 9 (b) satisfy $\omega=$ $0.021 \Omega=0.000147 \mathrm{~g}$, and the time for the system to reach the stationary state is about $1.6 \times 10^{5} / \mathrm{g}$. Nevertheless, the final fidelities of the desired steady state in Fig. 3(b) and Fig. 9(b) are almost the same. Hence, it is reasonable to conclude that the decrease of $\omega$ is at the cost of the convergence time of the system. Another important parameter in coupled cavities is the photon-hopping strength $J$, we plot the fidelity of state $\left|T_{1}\right\rangle$ versus time under the parameters $(g, \kappa$, $\gamma) / 2 \pi \sim(750,2.65,3.5) \mathrm{MHz}$ extracted from an experiment ${ }^{67}$ without feedback control in Fig. 10, from which one can see that the scheme has great robustness on the variety of $J$. In Fig. 11, without feedback control, we plot the fidelity of the desired state versus time with the parameters the same to Fig. 10 and $J=6 \mathrm{~g}$. Result shows that fidelity is higher than $97.2 \%$, which exceeds the values in the unitarydynamics-based schemes ${ }^{42-44}$. However, if we use another group of parameters $(g, \kappa, \gamma) / 2 \pi \sim(70,5,1) \mathrm{MHz}$ extracted from an experi- ment $^{68}$, the fidelity would be $91.4 \%$ without feedback control. That is, the fidelity depends on the parameter values. Moreover, Fig. 10 and Fig. 11 demonstrate that the presented scheme is also feasible without feedback control.

Compared with the dissipative scheme proposed in Ref. 8, the present one does not require $\kappa \gg \gamma$ since it takes advantage of both dissipative factors as resources to prepare entanglement. Compared with the scheme proposed in Ref. 9 which relies on the interference of photons emitted from the distant atoms, the present one could be implemented without detection of photons. Different with the unitary-dynamics-based three-dimensional entanglement preparation schemes ${ }^{42-44}$, the present one does not require precise time control and would be steady as time grows. Besides, the present scheme is independent of initial state. Moreover, with some specified parameters extracted from the experiment, the fidelity of our scheme could be higher than unitary-dynamics-based scheme. In a word, we could conclude that the overall performance of our scheme is better than that of the unitary-dynamics-based scheme.

In summary, we have proposed a scheme to prepare a threedimensional entangled state via using the dissipation. Spontaneous emission and cavity decay have been investigated to achieve the desired state, respectively and simultaneously. Moreover, we have investigated the influence of the feedback control on this scheme. Final numerical simulation based on one group of experiment parameters shows that our scheme could be feasible under current technology.

\section{Methods}

Effective operator method. Effective operator formalism for open quantum systems was pro-posed by Reiter and Sørensen in Ref. 47 and has been used widely in Refs. 13, $14,16,22,69-71$. First, they assume the open system consist of two distinct subspaces, one for the ground states and the other for the decaying excited states. Then, the couplings of these two subspaces should be perturbative. Furthermore, assuming that the dynamics of the system are Markovian such that the time evolution of the density operator $\rho$ can be described by a master equation of Lindblad form

$$
\dot{\rho}=-i[H, \rho]+\sum_{j}\left[L^{j} \rho L^{j \dagger}-\frac{1}{2}\left(L^{j \dagger} L^{j} \rho+\rho L^{j \dagger} L^{j}\right)\right],
$$

where $H$ is the Hamiltonian of the system and each of the Lindblad operators $L^{j}$ represents a source of decay which takes the system from the excited to the ground subspace. Through combining perturbation theory of the density operator and adiabatic elimination of the excited states they reduce the dynamics to an effective master equation involving only the ground-state manifold

$$
\dot{\rho}=-i\left[H_{\text {eff }}, \rho\right]+\sum_{j}\left[L_{\text {eff }}^{j} \rho L_{\text {eff }}^{j \dagger}-\frac{1}{2}\left(L_{\text {eff }}^{j \dagger} L_{\text {eff }}^{j} \rho+\rho L_{\text {eff }}^{j \dagger} L_{\text {eff }}^{j}\right)\right],
$$

with effective Hamilton and Lindblad operators

$$
\begin{aligned}
& H_{\text {eff }}=-\frac{1}{2}\left[V_{-} H_{\mathrm{NH}}^{-1} V_{+}+V_{-}\left(H_{\mathrm{NH}}^{-1}\right)^{\dagger} V_{+}\right]+H_{g}, \\
& L_{\text {eff }}^{j}=L^{j} H_{\mathrm{NH}}^{-1} V_{+},
\end{aligned}
$$

that only include the ground states. Here $V_{+}\left(V_{-}\right)$are the perturbative (de-)excitations of the system. $H_{g}$ is the ground-state Hamiltonian.

$H_{N H}=H_{0}-\frac{i}{2} \sum_{j} L^{\dagger \dagger} L^{j}$ is the non-Hermitian Hamiltonian with $H_{0}$ being the

Hamiltonian in the excited-state manifold. Since our scheme meets the conditions of the effective operator method, we used it to simplify the dynamic process of our system.

Obtaining the dominant dissipative channels. We first consider the spontaneousemission-based case. As we all know, the absolute value of the fraction would increase when the denominator decreases. Thus, if $\Delta \gg \gamma$,

$$
g^{2} \delta+\widetilde{J^{2}} \Delta=0
$$

would result in the dominant dissipative channels in Eq. (13) from Eq. (12). Through solving Eq. (22), we can get

$$
\delta=\left(g^{2}+\sqrt{g^{4}+4 J^{2} \Delta^{2}}\right) /(2 \Delta) .
$$




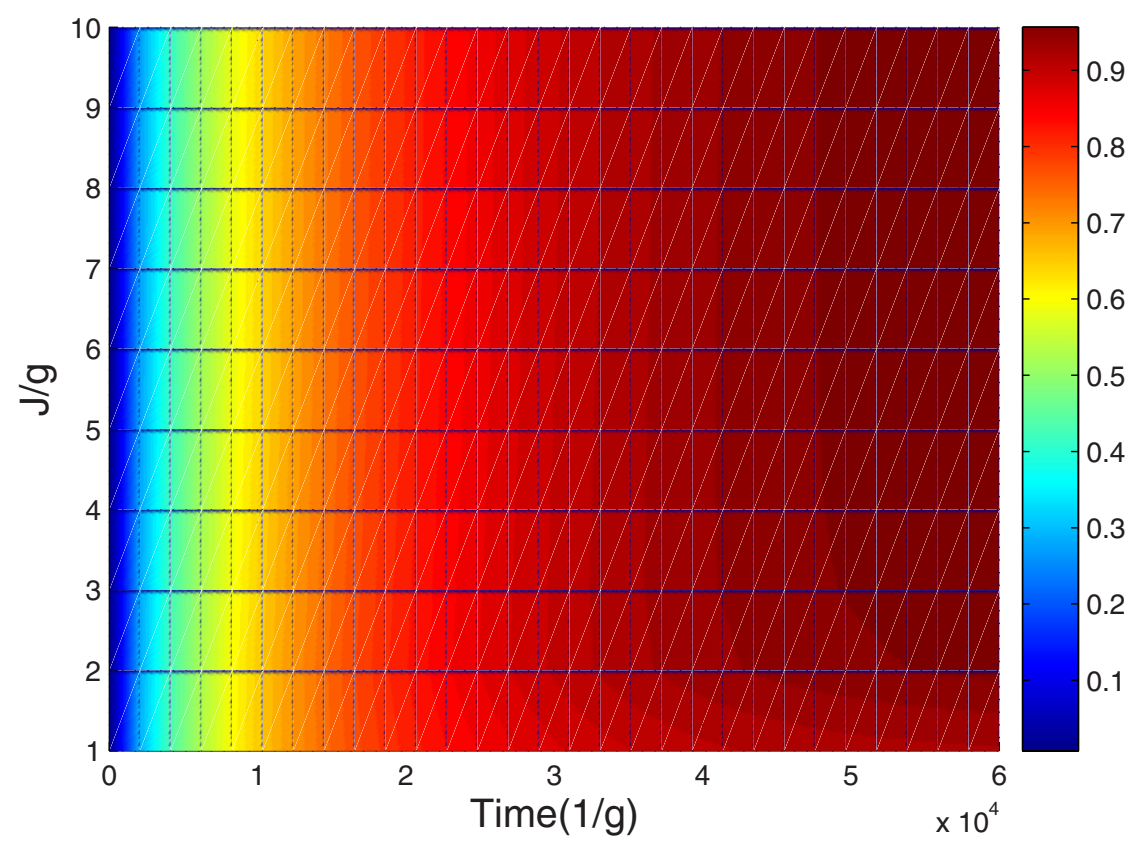

Figure $10 \mid$ Fidelity of $\left|T_{1}\right\rangle$ versus $J$ and time without feedback control. The other parameters are chosen as $\Omega=0.02 g, \omega=0.4 \Omega, \Delta=g$.

However, it should be noted that $2 g^{4}-3 g^{2} \delta \Delta-\widetilde{J}^{2} \Delta^{2}$ would be transformed to $-\left(g^{2} \delta+\widetilde{J^{2}} \Delta\right) \Delta$ when $\delta \Delta \simeq g^{2}$ and $\Delta=g$ (this condition has been used throughout this paper), and thus change the desired dominant dissipative channels. To prevent this, without loss of generality, we set

$$
\delta \Delta \geq \frac{4 g^{2}}{3} .
$$

Substituting Eq. (23) and $\Delta=g$ into Eq. (24), we can achieve

$$
J \geq \frac{2 g}{3} .
$$

The calculation process to obtain dominant dissipative channels of the cavity-decaybased case is similar to the spontaneous-emission-based case.

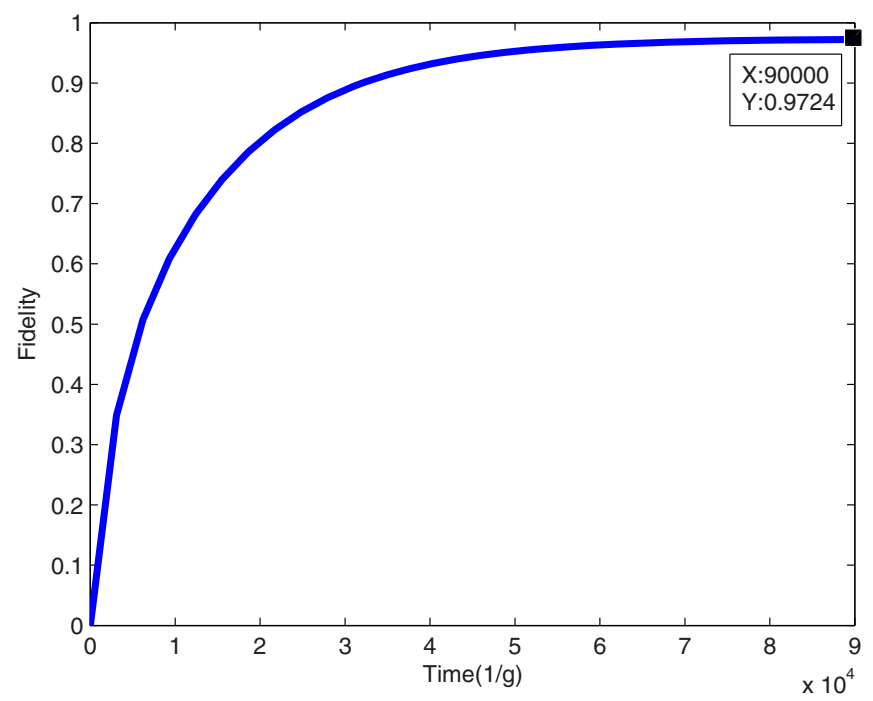

Figure $11 \mid$ Fidelity for generation of three-dimensional entangled state using one group of experimental cavity parameters without feedback control.
1. Steane, A. Multiple-particle interference and quantum error correction. Proc. $R$. Soc. A 452, 2551-2577 (1996).

2. Calderbank, A. R. \& Shor, P. W. Good quantum error-correcting codes exist. Phys. Rev. A 54, 1098-1105 (1996).

3. Chiaverini, J. et al. Realization of quantum error correction. Nature (London) 432, 602-605 (2004)

4. Palma, G. M., Suominen, K. A. \& Ekert, A. K. Quantum computers and dissipation. Proc. R. Soc. A 452, 567-584 (1996).

5. Duan, L.-M. \& Guo, G.-C. Preserving coherence in quantum computation by pairing quantum bits. Phys. Rev. Lett. 79, 1953-1956 (1997).

6. Zanardi, P. \& Rasetti, M. Noiseless quantum codes. Phys. Rev. Lett. 79, 3306-3309 (1997).

7. Lidar, D. A., Chuang, I. L. \& Whaley, K. B. Decoherence-free subspaces for quantum computation. Phys. Rev. Lett. 81, 2594-2597 (1998).

8. Plenio, M. B., Huelga, S. F., Beige, A. \& Knight, P. L. Cavity-loss-induced generation of entangled atoms. Phys. Rev. A 59, 2468-2475 (1999).

9. Cabrillo, C., Cirac, J. I., García-Fernández, P. \& Zoller, P. Creation of entangled states of distant atoms by interference. Phys. Rev. A 59, 1025-1033 (1999).

10. Diehl, S. et al. Quantum states and phases in driven open quantum systems with cold atoms. Nat. Phys. 4, 878-883 (2008).

11. Verstraete, F., Wolf, M. M. \& Cirac, J. I. Quantum computation and quantumstate engineering driven by dissipation. Nat. Phys. 5, 633-636 (2009)

12. Vacanti, G. \& Beige, A. Cooling atoms into entangled states. New J. Phys. 11, 083008 (2009).

13. Kastoryano, M. J., Reiter, F. \& Sørensen, A. S. Dissipative preparation of entanglement in optical cavities. Phys. Rev. Lett. 106, 090502 (2011).

14. Shen, L. T., Chen, X. Y., Yang, Z. B., Wu, H. Z. \& Zheng, S. B. Steady-state entanglement for distant atoms by dissipation in coupled cavities. Phys. Rev. A 84, 064302 (2011).

15. Shen, L. T., Chen, X. Y., Yang, Z. B., Wu, H. Z. \& Zheng, S. B. Distributed entanglement induced by dissipative bosonic media. Europhys. Lett. 99, 20003 (2012).

16. Reiter, F., Tornberg, L., Johansson, G. \& Sørensen, A. S. Steady-state entanglement of two superconducting qubits engineered by dissipation. Phys. Rev. A 88, 032317 (2013).

17. Leghtas, Z. et al. Stabilizing a Bell state of two superconducting qubits by dissipation engineering. Phys. Rev. A 88, 023849 (2013).

18. Bhaktavatsala Rao, D. D. \& Mølmer, K. Dark entangled steady states of interacting Rydberg atoms. Phys. Rev. Lett. 111, 033606 (2013).

19. Carr, A. W. \& Saffman, M. Preparation of entangled and antiferromagnetic states by dissipative Rydberg pumping. Phys. Rev. Lett. 111, 033607 (2013).

20. Busch, J. et al. Cooling atom-cavity systems into entangled states. Phys. Rev. A 84, 022316 (2011).

21. Lin, Y. et al. Dissipative production of a maximally entangled steady state of two quantum bits. Nature (London) 504, 415-418 (2013).

22. Shao, X. Q., Zheng, T. Y., Oh, C. H. \& Zhang, S. Dissipative creation of threedimensional entangled state in optical cavity via spontaneous emission. Phys. Rev. A 89, 012319 (2014). 
23. Shao, X. Q., You, J. B., Zheng, T. Y., Oh, C. H. \& Zhang, S. Stationary threedimensional entanglement via dissipative Rydberg pumping. Phys. Rev. A 89, 052313 (2014).

24. Dalla Torre, E. G., Otterbach, J., Demler, E., Vuletic, V. \& Lukin, M. D. Dissipative preparation of spin squeezed atomic ensembles in a steady state. Phys. Rev. Lett. 110, 120402 (2013).

25. Watanabe, G. \& Mäkelä, H. Dissipation-induced squeezing. Phys. Rev. A 85, 023604 (2012)

26. Caballar, R. C. F., Diehl, S., Mäkelä, H., Oberthaler, M. \& Watanabe, G. Dissipative preparation of phase- and number-squeezed states with ultracold atoms. Phys. Rev. A 89, 013620 (2014).

27. Song, J., Xia, Y. \& Song, H. S. One-step generation of cluster state by adiabatic passage in coupled cavities. Appl. Phys. Lett. 96, 071102 (2010).

28. Ogden, C. D., Irish, E. K. \& Kim, M. S. Dynamics in a coupled-cavity array. Phys. Rev. A 78, 063805 (2008).

29. Hartmann, M. J., Brandão, F. G. S. L. \& Plenio, M. B. Quantum many-body phenomena in coupled cavity arrays. Laser Photon. Rev. 2, 527-556 (2008).

30. Di Fidio, C. \& Vogel, W. Entanglement signature in the mode structure of a single photon. Phys. Rev. A 79, 050303(R) (2009).

31. Cho, J., Angelakis, D. G. \& Bose, S. Heralded generation of entanglement with coupled cavities. Phys. Rev. A 78, 022323 (2008).

32. Angelakis, D. G., Santos, M. F. \& Bose, S. Photon-blockade-induced Mott transitions and XY spin models in coupled cavity arrays. Phys. Rev. A 76, 031805(R) (2007).

33. Hartmann, M. J., Brandão, F. G. S. L. \& Plenio, M. B. Strongly interacting polaritons in coupled arrays of cavities. Nat. Phys. 2, 849-855 (2006).

34. Greentree, A. D., Tahan, C., Cole, J. H. \& Hollenberg, L. C. L. Quantum phase transitions of light. Nat. Phys. 2, 856-861 (2006).

35. Armani, D. K., Kippenberg, T. J., Spillane, S. M. \& Vahala, K. J. Ultra-high-Q toroid microcavity on a chip. Nature (London) 421, 925-928 (2003).

36. Durt, T., Cerf, N. J., Gisin, N. \& Żukowski, M. Security of quantum key distribution with entangled qutrits. Phys. Rev. A 67, 012311 (2003).

37. Durt, T., Kaszlikowski, D., Chen, J.-L. \& Kwek, L. C. Security of quantum key distributions with entangled qudits. Phys. Rev. A 69, 032313 (2004).

38. Kaszlikowski, D., Gnaciński, P., Żukowski, M., Miklaszewski, W. \& Zeilinger, A. Violations of local realism by two entangled n-dimensional systems are stronger than for two qubits. Phys. Rev. Lett. 85, 4418 (2000).

39. Mair, A., Vaziri, A., Weihs, G. \& Zeilinger, A. Entanglement of the orbital angular momentum states of photons. Nature (London) 412, 313 (2001).

40. Vaziri, A., Weihs, G. \& Zeilinger, A. Experimental two-photon, three-dimensional entanglement for quantum communication. Phys. Rev. Lett. 89, 240401 (2002).

41. Zou, X. B., Pahlke, K. \& Mathis, W. Generation of an entangled state of two threelevel atoms in cavity QED. Phys. Rev. A 67, 044301 (2003).

42. Ye, S. Y., Zhong, Z. R. \& Zheng, S. B. Deterministic generation of threedimensional entanglement for two atoms separately trapped in two optical cavities. Phys. Rev. A 77, 014303 (2008).

43. Lü, X. Y., Liu, J. B., Ding, C. L. \& Li, J. H. Dispersive atom-field interaction scheme for three-dimensional entanglement between two spatially separated atoms. Phys. Rev. A 78, 032305 (2008).

44. Li, W. A. \& Huang, G. Y. Deterministic generation of a three-dimensional entangled state via quantum Zeno dynamic. Phys. Rev. A 83, 022322 (2011).

45. Busch, J. et al. Cooling atom-cavity systems into entangled states. Phys. Rev. A $\mathbf{8 4}$ 022316 (2011)

46. Shen, L. T., Chen, X. Y., Yang, Z. B., Wu, H. Z. \& Zheng, S. B. Cooling distant atoms into steady entanglement via coupled cavities. Quantu. Inf. Comput. 13, 281-289 (2013).

47. Reiter, F. \& Sørensen, A. S. Effective operator formalism for open quantum systems. Phys. Rev. A 85, 032111 (2012).

48. Knight, P. L. Quantum fluctuations and squeezing in the interaction of an atom with a single field mode. Phys. Scr. T12, 51-55 (1986)

49. Gerry, C. C. Degenerate Raman coupled model interacting with two quantized cavity fields. Phys. Lett. A 161, 9-12 (1991).

50. Puri, R. R. \& Agarwal, G. S. Exact density matrix for the degenerate-Ramancoupled model in the presence of collisions. Phys. Rev. A 45, 5073-5077 (1992).

51. Xu, L., Luo, Z. F. \& Zhang, Z. M. Validity of the effective Hamiltonian for the degenerate Raman process. J. Phys. B: At. Mol. Opt. Phys. 27, 1649-1656 (1994).

52. Xu, L. \& Zhang, Z. M. Modified effective Hamiltonian for degenerate Raman process. Z. Phys. B 95, 507-610 (1994).

53. Togan, E. et al. Quantum entanglement between an optical photon and a solidstate spin qubit. Nature (London) 466, 730-734 (2010).
54. Geremia, J. M., Stockton, J. K. \& Mabuchi, H. Real-time quantum feedback control of atomic spin-squeezing. Science 304, 270-273 (2004).

55. Bushev, P. et al. Feedback cooling of a single trapped ion. Phys. Rev. Lett. 96, 043003 (2006).

56. Shabani, A. \& Jacobs, K. Locally optimal control of quantum systems with strong feedback. Phys. Rev. Lett. 101, 230403 (2008).

57. Wiseman, H. M. \& Milburn, G. J. Quantum measurement and control (Cambridge University Press, Cambridge, 2010).

58. Jacobs, K. Feedback control using only quantum back-action. New J. Phys. 12, 043005 (2010).

59. Wang, J., Wiseman, H. M. \& Milburn, G. J. Dynamical creation of entanglement by homodyne-mediated feedback. Phys. Rev. A 71, 042309 (2005).

60. Carvalho, A. R. R. \& Hope, J. J. Stabilizing entanglement by quantum-jump-based feedback. Phys. Rev. A 76, 010301(R) (2007).

61. Carvalho, A. R. R., Reid, A. J. S. \& Hope, J. J. Controlling entanglement by direct quantum feedback. Phys. Rev. A 78, 012334 (2008).

62. Stevenson, R. N., Hope, J. J. \& Carvalho, A. R. R. Engineering steady states using jump-based feedback for multipartite entanglement generation. Phys. Rev. A 84, 022332 (2011).

63. Shao, X. Q., Zheng, T. Y. \& Zhang, S. Engineering steady three-atom singlet states via quantum-jump-based feedback. Phys. Rev. A 85, 042308 (2012).

64. Imamoglu, A. et al. Quantum information processing using quantum dot spins and cavity QED. Phys. Rev. Lett. 83, 4204-4207 (1999).

65. James, D. F. V. \& Jerke, J. Effective Hamiltonian theory and its applications in quantum information. Can. J. Phys. 85, 625-632 (2007).

66. Gamel, O. \& James, D. F. V. Time-averaged quantum dynamics and the validity of the effective Hamiltonian model. Phys. Rev. A 82, 052106 (2010).

67. Spillane, S. M. et al. Ultrahigh-Q toroidal microresonators for cavity quantum electrodynamics. Phys. Rev. A 71, 013817 (2005).

68. Dayan, B. et al. A photon turnstile dynamically regulated by one atom. Science 319, 1062-1065 (2008).

69. Reiter, F., Kastoryano, M. J. \& Sørensen, A. S. Driving two atoms in an optical cavity into an entangled steady state using engineered decay. New J. Phys. 14, 053022 (2012).

70. Sweke, R., Sinayskiy, I. \& Petruccione, F. Dissipative preparation of large W states in optical cavities. Phys. Rev. A 87, 042323 (2013).

71. Sweke, R., Sinayskiy, I. \& Petruccione, F. Dissipative preparation of generalized Bell states. J. Phys. B: At. Mol. Opt. Phys. 46, 104004 (2013).

\section{Acknowledgments}

This work was supported by the National Natural Science Foundation of China under Grant No. 11264042, No. 11465020, No. 11204028, and No. 61465013. X. Q. Shao was also supported by Fundamental Research Funds for the Central Universities under Grant No. 12SSXM001, and the Government of China through CSC. H. F. Wang was also supported by Chun Miao Excellent Talents of Jilin Provincial Department of Education under Grant No. 201316

\section{Author contributions}

S.L. carried out all calculations under the guidance of X.Q., H.F. and S.Z. All authors contributed to the interpretation of the work and the writing of the manuscript. All authors reviewed the manuscript.

\section{Additional information}

Competing financial interests: The authors declare no competing financial interests.

How to cite this article: Su, S.-L., Shao, X.-Q., Wang, H.-F. \& Zhang, S. Preparation of three-dimensional entanglement for distant atoms in coupled cavities via atomic spontaneous emission and cavity decay. Sci. Rep. 4, 7566; DOI:10.1038/srep07566 (2014)

This work is licensed under a Creative Commons Attribution-NonCommercialNoDerivs 4.0 International License. The images or other third party material in this article are included in the article's Creative Commons license, unless indicated otherwise in the credit line; if the material is not included under the Creative Commons license, users will need to obtain permission from the license holder in order to reproduce the material. To view a copy of this license, visit http:// creativecommons.org/licenses/by-nc-nd/4.0/ 